\title{
Israel: Statistical Appendix
}

This Statistical Appendix for Israel was prepared by a staff team of the International Monetary Fund as background documentation for the periodic consultation with the member country. It is based on the information available at the time it was completed on March 3, 2004. The views expressed in this document are those of the staff team and do not necessarily reflect the views of the government of Israel or the Executive Board of the IMF.

The policy of publication of staff reports and other documents by the IMF allows for the deletion of market-sensitive information.

To assist the IMF in evaluating the publication policy, reader comments are invited and may be sent by e-mail to publicationpolicy@imf.org.

Copies of this report are available to the public from

International Monetary Fund $\bullet$ Publication Services

700 19th Street, N.W. • Washington, D.C. 20431

Telephone: (202) 6237430 • Telefax: (202) 6237201

E-mail: publications@imf.org • Internet: http://www.imf.org

Price: $\$ 15.00$ a copy

\section{International Monetary Fund} Washington, D.C. 



\title{
INTERNATIONAL MONETARY FUND
}

\author{
ISRAEL \\ Statistical Appendix \\ Prepared by Gil Mehrez \\ Approved by European Department
}

March 3, 2004

Contents

Page

1. GDP by Expenditure Components in Current Prices, 1997-2003 ...................................3

2. GDP by Expenditure Components in Constant Prices, 1997-2003 …………..................

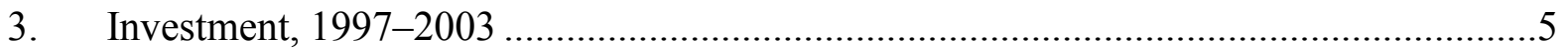

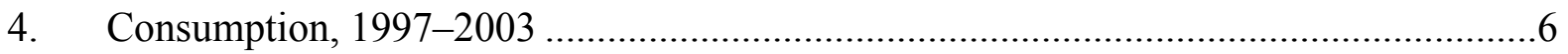

5. Gross Private Income and Savings, 1997-2003 ……….............................................

6. National Savings, Foreign Savings, and Investment, 1997-2003 ................................. 8

7. Composition and Growth of the Industrial Production, 1997-2003 ………...................9

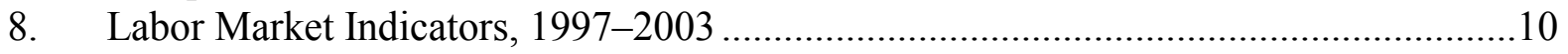

9. Employment and Labor Input of Israelis by Industry, 1997-2003 ..............................11

10. Real Wages, Labor Costs, and Productivity, 1997-2003 ………..................................12

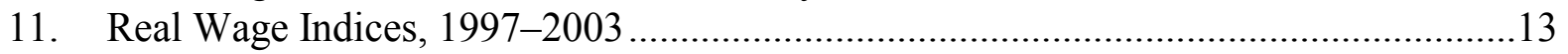

12. Consumer Price Index and its Main Components, 1999-2003 ….................................14

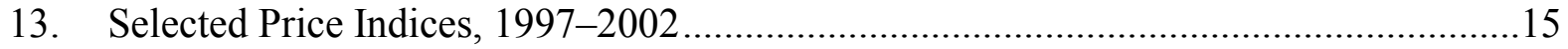

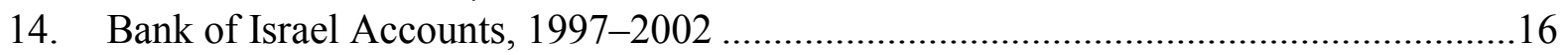

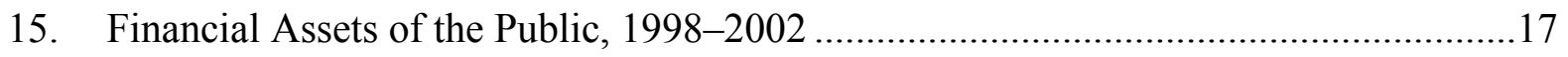

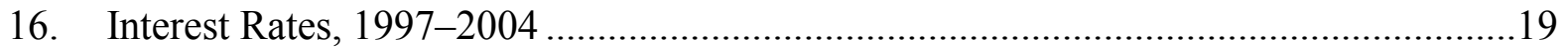

17. Interest Rates on Various Types of Credit and the Public's Assets, 1998-2003 ...........20

18. Reserve Requirements on Deposits and Interest Brackets, 1994-2003 .........................21

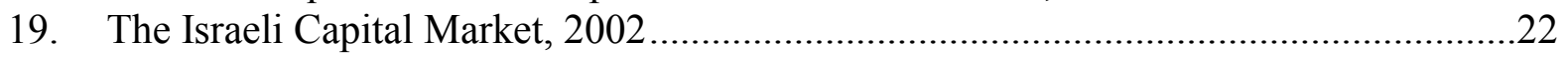

20. Principal Stock Market Indicators, 1997-2002 .........................................................23

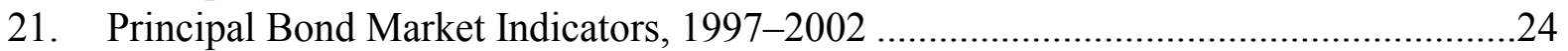

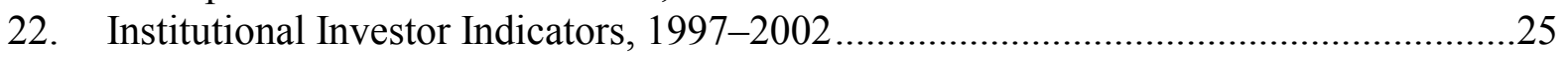

23. State Budget Balance and Financing, 1999-2003 …….............................................26

24. State Budget Revenue, 1999-2003 ......................................................................2

25. State Budget Expenditure (Economic Classification) 1999-2003 .................................28

26. State Budget Expenditure (Functional Classification), 1999-2003 .............................29

27. General Government Balance and Financial (National Accounts), 1997-2003 ............30

28. General Government Receipts (National Accounts), 1997-2003 .................................31 
29. General Government Expenditure (National Accounts), 1997-2003 .........................32

30. Structure of General Government Finances (National Accounts), 1997-2003 .............33

31. Currency Basket of the Israeli Sheqel, 1994-2003 ....................................................34

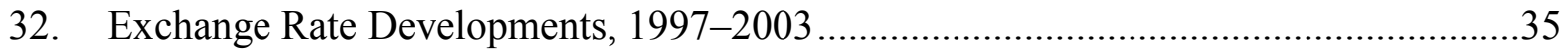

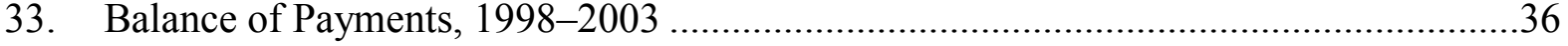

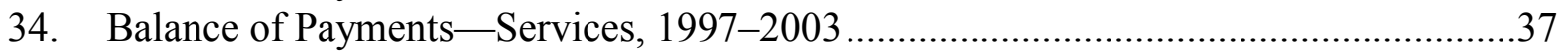

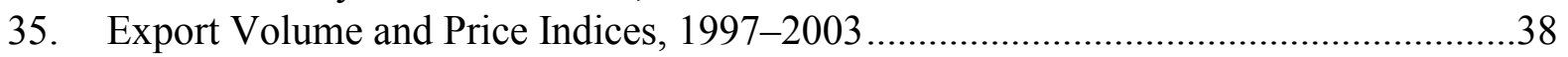

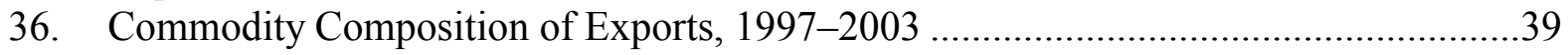

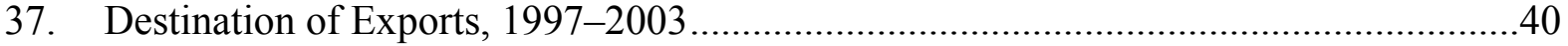

38. Civilian Import Volume and Price Indicators, 2001-03 .............................................41

39. Commodity Composition of Civilian Imports, 1997-2003 .........................................42

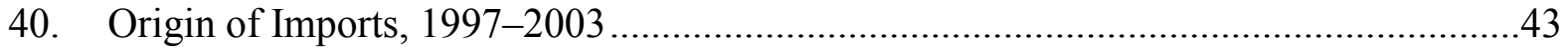

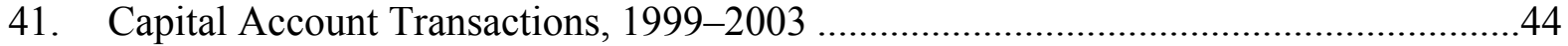

42. Indicators of External Indebtedness, 1997-2003 ….................................................45

43. Assets and Liabilities in Foreign Currency, 1997-2003 .........................................46

44. Official Gold and Convertible Foreign Exchange Reserves, 1997-2003 ...................47 
Table 1. Israel: GDP by Expenditure Components in Current Prices, 1997-2003 1/

\begin{tabular}{|c|c|c|c|c|c|c|c|}
\hline & 1997 & 1998 & 1999 & 2000 & 2001 & 2002 & 2003 \\
\hline & \multicolumn{7}{|c|}{ (In millions of new sheqalim; current prices) } \\
\hline GDP & 356,055 & 393,104 & 429,542 & 468,144 & 474,043 & 491,260 & 498,661 \\
\hline Domestic demand & 392,564 & 423,006 & 463,002 & 491,684 & 510,346 & 534,190 & 532,505 \\
\hline Consumption & 306,206 & 334,309 & 364,432 & 393,723 & 414,911 & 444,733 & 451,740 \\
\hline Private & 203,431 & 222,531 & 242,684 & 263,762 & 275,957 & 291,620 & 299,795 \\
\hline Public & 102,775 & 111,779 & 121,748 & 129,961 & 138,954 & 153,113 & 151,945 \\
\hline Gross domestic capital formation & 86,357 & 88,697 & 98,571 & 97,961 & 95,434 & 89,457 & 80,765 \\
\hline Fixed capital formation & 83,947 & 85,808 & 92,169 & 92,846 & 89,136 & 87,234 & 86,773 \\
\hline Increase in stocks & 2,411 & 2,889 & 6,402 & 5,115 & 6,298 & 2,224 & $-6,008$ \\
\hline Foreign balance & $-36,509$ & $-29,902$ & $-33,460$ & $-23,540$ & $-36,303$ & $-42,930$ & $-33,844$ \\
\hline Exports of goods and nonfactor services & 109,236 & 124,466 & 155,616 & 189,127 & 168,107 & 181,962 & 185,153 \\
\hline \multirow[t]{2}{*}{ Imports of goods and nonfactor services } & 145,745 & 154,368 & 189,076 & 212,667 & 204,410 & 224,892 & 218,997 \\
\hline & \multicolumn{7}{|c|}{ (In percent of GDP; current prices) } \\
\hline GDP & 100.0 & 100.0 & 100.0 & 100.0 & 100.0 & 100.0 & 100.0 \\
\hline Domestic demand & 110.3 & 107.6 & 107.8 & 105.0 & 107.7 & 108.7 & 106.8 \\
\hline Consumption & 86.0 & 85.0 & 84.8 & 84.1 & 87.5 & 90.5 & 90.6 \\
\hline Private & 57.1 & 56.6 & 56.5 & 56.3 & 58.2 & 59.4 & 60.1 \\
\hline Public & 28.9 & 28.4 & 28.3 & 27.8 & 29.3 & 31.2 & 30.5 \\
\hline Gross domestic capital formation & 24.3 & 22.6 & 22.9 & 20.9 & 20.1 & 18.2 & 16.2 \\
\hline Fixed capital formation & 23.6 & 21.8 & 21.5 & 19.8 & 18.8 & 17.8 & 17.4 \\
\hline Increase in stocks & 0.7 & 0.7 & 1.5 & 1.1 & 1.3 & 0.5 & -1.2 \\
\hline Foreign balance & -10.3 & -7.6 & -7.8 & -5.0 & -7.7 & -8.7 & -6.8 \\
\hline Exports of goods and nonfactor services & 30.7 & 31.7 & 36.2 & 40.4 & 35.5 & 37.0 & 37.1 \\
\hline Imports of goods and nonfactor services & 40.9 & 39.3 & 44.0 & 45.4 & 43.1 & 45.8 & 43.9 \\
\hline
\end{tabular}

Source: Central Bureau of Statistics, Monthly Bulletin of Statistics.

1/ Preliminary estimates of the Central Bureau of Statistics. 
Table 2. Israel: GDP by Expenditure Components in Constant Prices, 1997-2003 1/ 2 /

\begin{tabular}{|c|c|c|c|c|c|c|c|}
\hline & 1997 & 1998 & 1999 & 2000 & 2001 & 2002 & 2003 \\
\hline & \multicolumn{7}{|c|}{ (In millions of new sheqalim; constant 2000 prices) } \\
\hline GDP & 410,708 & 424,125 & 435,348 & 468,144 & 463,906 & 460,221 & 465,256 \\
\hline Domestic demand & 445,281 & 453,376 & 473,153 & 491,684 & 499,603 & 496,253 & 489,872 \\
\hline Consumption & 346,874 & 359,089 & 372,462 & 393,725 & 406,481 & 414,586 & 417,588 \\
\hline Private & 225,819 & 235,413 & 245,246 & 263,762 & 272,114 & 272,501 & 275,628 \\
\hline Public & 121,055 & 123,675 & 127,216 & 129,963 & 134,367 & 142,084 & 141,960 \\
\hline Gross domestic capital formation & 98,762 & 94,495 & 100,843 & 97,959 & 93,121 & 81,613 & 72,284 \\
\hline Fixed capital formation & 94,778 & 91,177 & 91,301 & 92,845 & 88,398 & 80,262 & 78,056 \\
\hline Increase in stocks & 3,984 & 3,317 & 9,542 & 5,114 & 4,724 & 1,351 & $-5,772$ \\
\hline Foreign balance & $-34,167$ & $-28,753$ & $-36,972$ & $-23,540$ & $-35,697$ & $-36,049$ & $-25,076$ \\
\hline Exports of goods and nonfactor services & 128,044 & 136,190 & 152,520 & 189,127 & 167,317 & 162,336 & 166,394 \\
\hline \multirow[t]{2}{*}{ Imports of goods and nonfactor services } & 162,211 & 164,943 & 189,492 & 212,667 & 203,014 & 198,385 & 191,470 \\
\hline & \multicolumn{7}{|c|}{ (Annual percentage change) } \\
\hline GDP & 3.0 & 3.3 & 2.6 & 7.5 & -0.9 & -0.8 & 1.1 \\
\hline Domestic demand & 1.6 & 1.8 & 4.4 & 3.9 & 1.6 & -0.7 & -1.3 \\
\hline Consumption & 2.6 & 3.5 & 3.7 & 5.7 & 3.2 & 2.0 & 0.7 \\
\hline Private & 2.9 & 4.2 & 4.2 & 7.6 & 3.2 & 0.1 & 1.1 \\
\hline Public & 1.9 & 2.2 & 2.9 & 2.2 & 3.4 & 5.7 & -0.1 \\
\hline Gross domestic capital formation & -1.6 & -4.3 & 6.7 & -2.9 & -4.9 & -12.4 & -11.4 \\
\hline Fixed capital formation & -1.0 & -3.8 & 0.1 & 1.7 & -4.8 & -9.2 & -2.7 \\
\hline Exports of goods and nonfactor services & 8.6 & 6.4 & 12.0 & 24.0 & -11.5 & -3.0 & 2.5 \\
\hline Imports of goods and nonfactor services & 3.3 & 1.7 & 14.9 & 12.2 & -4.5 & -2.3 & -3.5 \\
\hline
\end{tabular}

Source: Central Bureau of Statistics, Monthly Bulletin of Statistics.

1/ Estimates in 2000 prices derived by chaining each categories growth rate computed at different base year prices, hence totals do not reflect the sum of their components.

2/ Preliminary estimates of the Central Bureau of Statistics. 


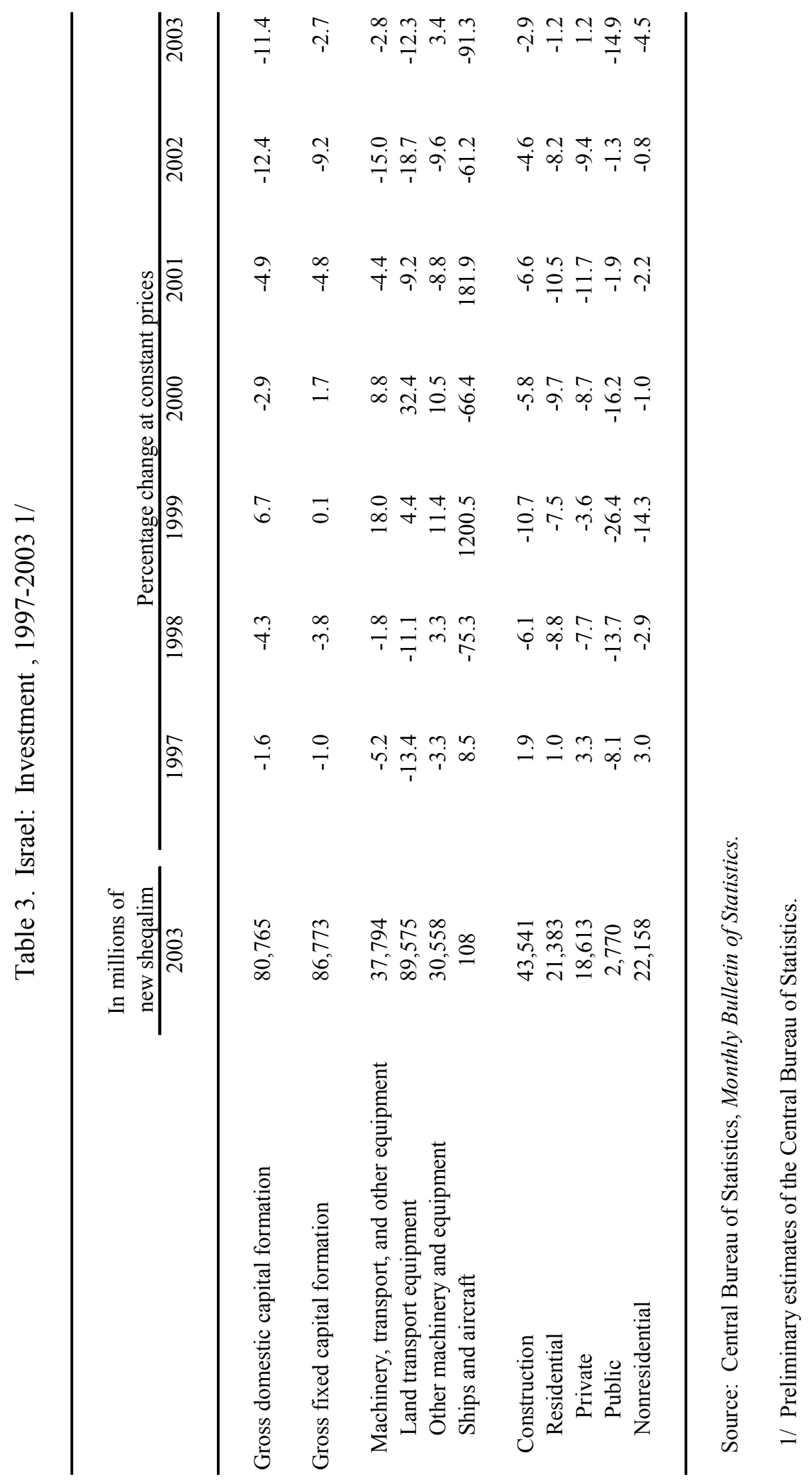




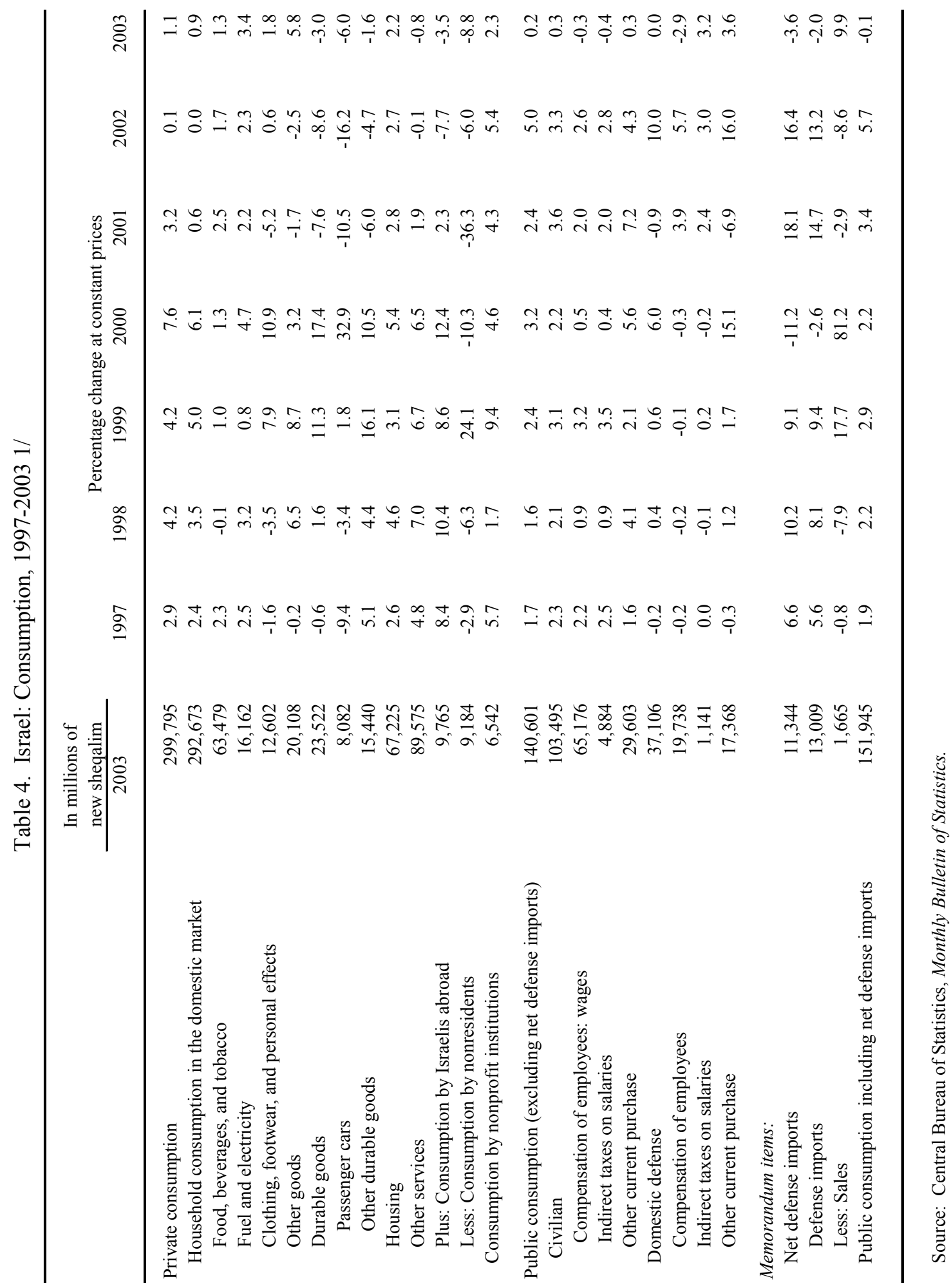


Table 5. Israel: Gross Private Income and Savings, 1997-2003 1/

\begin{tabular}{|c|c|c|c|c|c|c|c|}
\hline & 1997 & 1998 & 1999 & 2000 & 2001 & 2002 & 2003 \\
\hline & \multicolumn{7}{|c|}{ (In millions of new sheqalim; at current prices) } \\
\hline GNP at market prices & 347,481 & 380,799 & 414,130 & 442,929 & 457,584 & 477,993 & 488,542 \\
\hline \multicolumn{8}{|l|}{ Plus: } \\
\hline Subsidies on domestic production & 2,845 & 2,854 & 2,989 & 3,214 & 3,337 & 2,953 & 3,296 \\
\hline Subsidies on government loans & 189 & 138 & 66 & 47 & 44 & 25 & 24 \\
\hline \multicolumn{8}{|l|}{ Less: } \\
\hline Indirect taxes on domestic production & 49,340 & 53,466 & 58,579 & 59,889 & 62,157 & 67,908 & 68,161 \\
\hline \multicolumn{8}{|l|}{ Equals: } \\
\hline GNP at factor cost & 301,175 & 330,325 & 358,606 & 386,301 & 398,808 & 413,063 & 423,701 \\
\hline \multicolumn{8}{|l|}{ Plus: } \\
\hline Net private transfers from abroad & 7,897 & 8,847 & 9,483 & 9,493 & 9,811 & 11,097 & 11,308 \\
\hline $\begin{array}{l}\text { Net transfers to domestic households } \\
\text { and private nonprofit institutions }\end{array}$ & 54,760 & 61,736 & 66,082 & 73,106 & 79,223 & 79,708 & 83,941 \\
\hline \multicolumn{8}{|l|}{ Less: } \\
\hline $\begin{array}{l}\text { Public sector income from property } \\
\text { and entrepreneurship }\end{array}$ & 2,652 & 4,538 & 475 & 276 & 2,705 & 8,054 & 4,528 \\
\hline Public sector consumption of fixed capital & 4,952 & 5,589 & 6,314 & 6,711 & 7,129 & 7,996 & 8,716 \\
\hline \multicolumn{8}{|l|}{ Equals: } \\
\hline Private income & 356,228 & 390,781 & 427,383 & 461,913 & 478,008 & 487,818 & 505,706 \\
\hline \multicolumn{8}{|l|}{ Less: } \\
\hline Direct taxes 2/ & 71,744 & 78,173 & 85,154 & 102,455 & 105,105 & 101,996 & 99,298 \\
\hline \multicolumn{8}{|l|}{ Equals: } \\
\hline Gross private disposable income & 284,484 & 312,608 & 342,229 & 359,458 & 372,903 & 385,822 & 406,408 \\
\hline \multicolumn{8}{|l|}{ Less: } \\
\hline Private consumption & 203,431 & 222,531 & 242,684 & 263,762 & 275,957 & 291,620 & 299,795 \\
\hline \multicolumn{8}{|l|}{ Equals: } \\
\hline \multirow[t]{2}{*}{ Private saving } & 81,053 & 90,078 & 99,545 & 95,696 & 96,946 & 94,202 & 106,613 \\
\hline & \multicolumn{7}{|c|}{ (percent of gross private disposable income) } \\
\hline Private consumption & 71.5 & 71.2 & 70.9 & 73.4 & 74.0 & 75.6 & 73.8 \\
\hline Private savings & 28.5 & 28.8 & 29.1 & 26.6 & 26.0 & 24.4 & 26.2 \\
\hline
\end{tabular}

Sources: Central Bureau of Statistics, Current Briefings in Statistics; and data provided by the Bank of Israel.

1/ Preliminary estimates of the Central Bureau of Statistics.

2/ Including contribution to National Insurance Institute. 
Table 6. Israel: National Savings, Foreign Savings, and Investment, 1997-2003 1/

\begin{tabular}{|c|c|c|c|c|c|c|c|}
\hline & 1997 & 1998 & 1999 & 2000 & 2001 & 2002 & 2003 \\
\hline & \multicolumn{7}{|c|}{ (percent of total income) $2 /$} \\
\hline Gross national savings & 20.0 & 20.4 & 20.3 & 19.5 & 17.6 & 15.8 & 16.0 \\
\hline General government & -1.2 & -1.1 & -1.5 & -0.1 & -1.8 & -2.1 & -3.9 \\
\hline Private sector & 21.3 & 21.5 & 21.8 & 19.6 & 19.3 & 17.9 & 19.9 \\
\hline Foreign savings & 2.6 & 0.8 & 1.3 & 0.6 & 1.5 & 1.2 & -1.0 \\
\hline Current account & -2.6 & -0.8 & -1.3 & -0.6 & -1.5 & -1.2 & 1.0 \\
\hline Civilian trade balance & -5.7 & -4.1 & -4.4 & -3.7 & -4.4 & -4.2 & -2.1 \\
\hline Unilateral transfers & 3.7 & 3.8 & 3.6 & 3.5 & 3.5 & 3.1 & 3.4 \\
\hline Transfers on capital account & -0.7 & -0.5 & -0.5 & -0.4 & -0.6 & -0.1 & -0.3 \\
\hline Gross investment & 22.6 & 21.2 & 21.6 & 20.1 & 19.0 & 17.0 & 15.0 \\
\hline Inventories & 0.6 & 0.7 & 1.4 & 1.0 & 1.3 & 0.4 & -1.2 \\
\hline Fixed residential & 7.3 & 6.3 & 5.7 & 4.9 & 4.3 & 3.9 & 4.0 \\
\hline \multirow[t]{2}{*}{ Fixed nonresidential } & 14.7 & 14.2 & 14.6 & 14.2 & 13.5 & 12.7 & 12.2 \\
\hline & \multicolumn{7}{|c|}{ (percent of GDP) } \\
\hline Gross national savings & 21.4 & 21.7 & 21.6 & 20.3 & 18.6 & 16.9 & 17.3 \\
\hline General government & -1.3 & -1.2 & -1.6 & -0.1 & -1.9 & -2.3 & -4.2 \\
\hline Private sector & 22.8 & 22.9 & 23.1 & 20.4 & 20.5 & 19.2 & 21.5 \\
\hline Foreign savings & 2.8 & 0.8 & 1.4 & 0.6 & 1.5 & 1.3 & -1.1 \\
\hline Current account & -2.8 & -0.8 & -1.4 & -0.6 & -1.5 & -1.3 & 1.1 \\
\hline Civilian trade balance & -6.1 & -4.3 & -4.7 & -3.9 & -4.7 & -4.5 & -2.2 \\
\hline Unilateral transfers & 4.0 & 4.1 & 3.8 & 3.7 & 3.7 & 3.3 & 3.7 \\
\hline Transfers on capital account & -0.7 & -0.6 & -0.5 & -0.4 & -0.6 & -0.1 & -0.4 \\
\hline Gross investment & 24.3 & 22.6 & 22.9 & 20.9 & 20.1 & 18.2 & 16.2 \\
\hline Inventories & 0.7 & 0.7 & 1.5 & 1.1 & 1.3 & 0.5 & -1.3 \\
\hline Fixed residential & 7.8 & 6.7 & 6.0 & 5.1 & 4.5 & 4.2 & 4.3 \\
\hline Fixed nonresidential & 15.8 & 15.1 & 15.5 & 14.8 & 14.3 & 13.5 & 13.2 \\
\hline
\end{tabular}

Source: Data provided by the Bank of Israel.

1/ Preliminary estimates of the Central Bureau of Statistics.

2/ Total income defined as GNP plus unilateral transfers from abroad. 
Table 7. Israel: Composition and Growth of the Industrial Production, 1997-2003 2/

\begin{tabular}{|c|c|c|c|c|c|c|c|}
\hline & $\frac{\text { Weight }}{2002}$ & 1998 & 1999 & 2000 & 2001 & 2002 & 2003 \\
\hline & & \multicolumn{6}{|c|}{ (Percentage change at constant prices) } \\
\hline Total industrial production $1 /$ & 100.0 & 2.9 & 1.4 & 10.0 & -5.0 & -1.9 & -1.3 \\
\hline Food, beverages, and tobacco & 11.4 & 0.8 & 1.4 & 0.1 & -1.1 & -1.4 & -2.9 \\
\hline Textiles, clothing and leather & 4.4 & -0.1 & 1.5 & -3.1 & -4.4 & -4.7 & -8.5 \\
\hline Wood, furniture, paper and printing & 9.3 & -1.4 & -1.8 & 1.3 & -7.0 & -2.1 & -3.4 \\
\hline Chemicals, rubber, and plastics & 19.1 & 9.3 & -0.7 & 3.4 & 4.9 & 13.2 & 4.9 \\
\hline Nonmetallic minerals & 2.4 & -12.3 & -8.2 & -8.3 & -7.0 & -5.6 & -7.9 \\
\hline Basic metals and metal products & 11.9 & -1.8 & -2.9 & 7.7 & -4.8 & -2.6 & -4.0 \\
\hline Machinery and equipment (incl. Electric) & 5.5 & 3.8 & 2.2 & 8.6 & -8.7 & -5.5 & -3.3 \\
\hline Transport equipment & 6.1 & 6.6 & 3.1 & 4.9 & 1.4 & 1.4 & 3.6 \\
\hline Electronic equipment & 8.1 & 11.8 & 8.8 & 16.0 & -16.8 & -14.6 & -8.4 \\
\hline Jewelry and miscellaneous articles & 1.5 & -4.0 & -9.8 & 1.2 & 0.4 & -1.6 & -4.1 \\
\hline
\end{tabular}

Sources: Central Bureau of Statistics, Monthly Bulletin of Statistics; and data provided by the Bank of Israel.

1/ Includes sectors not listed below.

2/ Preliminary estimates of the Central Bureau of Statistics. 


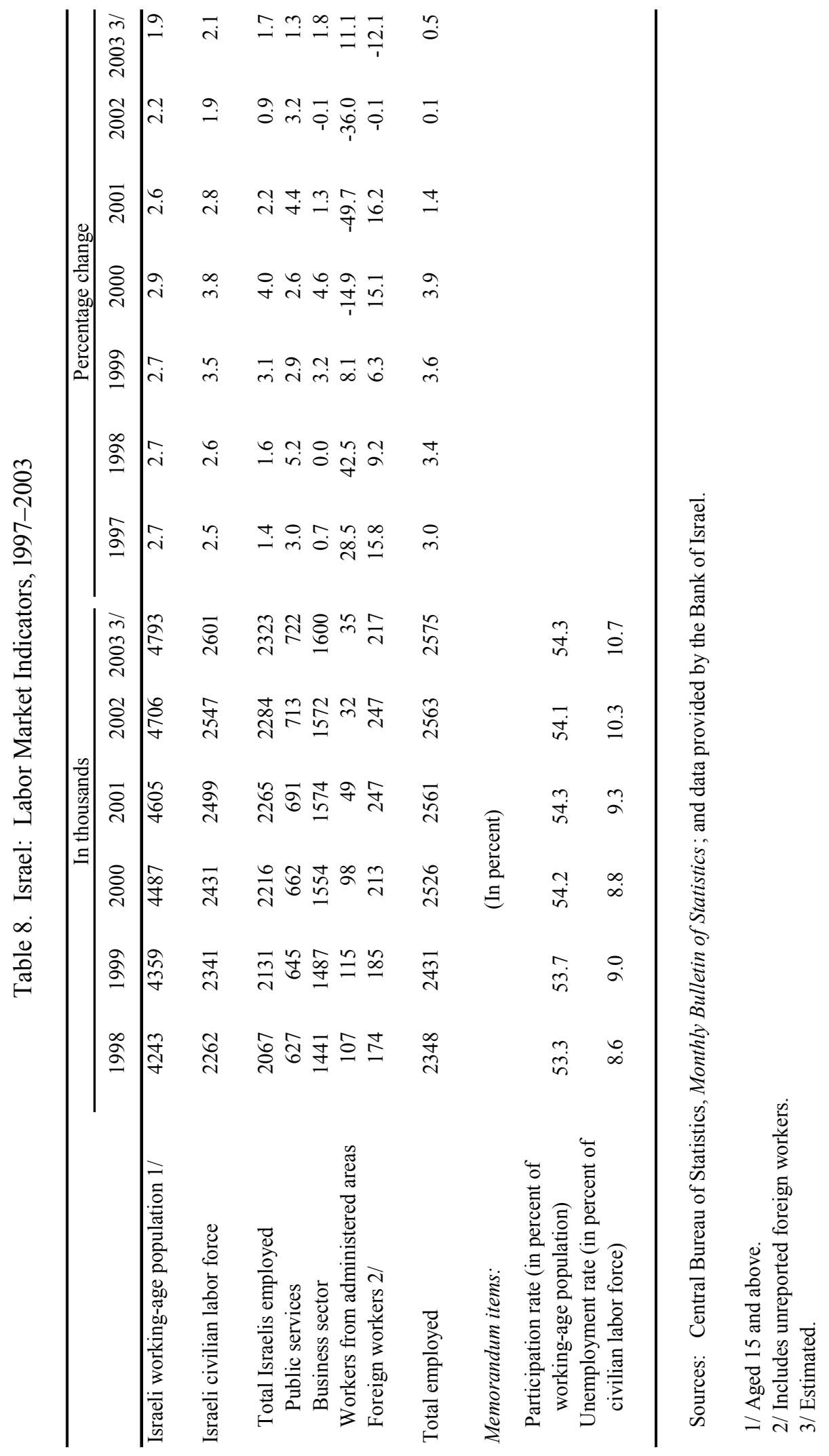


Table 9. Israel: Employment and Labor Input of Israelis by Industry, 1997-2003 1/

\begin{tabular}{|c|c|c|c|c|c|c|c|c|}
\hline & $20032 /$ & 1997 & 1998 & 1999 & 2000 & 2001 & 2002 & $20032 /$ \\
\hline & In thousands & \multicolumn{7}{|c|}{ Annual percentage change } \\
\hline \multicolumn{9}{|l|}{ Employed persons } \\
\hline Total 3/ & 2319.4 & 1.4 & 1.6 & 3.1 & 4.0 & 2.2 & 0.9 & 2.0 \\
\hline Agriculture & 41.9 & -4.2 & -1.2 & 4.4 & -3.3 & -10.2 & -0.4 & -4.0 \\
\hline Industry & 381.8 & -1.7 & -2.3 & 1.0 & 1.7 & -0.6 & -3.8 & 2.4 \\
\hline Public utilities & 18.4 & 2.3 & 7.8 & -4.1 & 0.5 & 1.1 & 0.0 & -11.0 \\
\hline Construction & 129.5 & -2.5 & -10.5 & -8.6 & -3.2 & 0.3 & 1.4 & 8.4 \\
\hline Commerce & 305.5 & 3.0 & 2.8 & 3.3 & 5.0 & 1.4 & 4.2 & -0.4 \\
\hline Hotels and restaurants & 91.8 & -0.4 & 7.4 & 11.9 & 12.7 & -5.0 & -3.1 & -0.2 \\
\hline Transport, storage, and communication & 149.9 & 0.1 & -0.5 & 10.7 & 7.0 & 3.0 & -2.0 & 2.4 \\
\hline Banking, insurance, and finance & 77.5 & 8.9 & -0.5 & 1.2 & -0.8 & 2.2 & 1.3 & 2.8 \\
\hline Business services & 293.8 & 5.6 & 5.8 & 3.7 & 15.1 & 7.2 & -0.9 & 7.5 \\
\hline Community, social, and personal services & 114.2 & 1.2 & -0.3 & 0.4 & 7.3 & 1.5 & 2.9 & 2.7 \\
\hline Civil service & 129.9 & 5.3 & -0.9 & 3.4 & 3.0 & 5.6 & 4.0 & -4.0 \\
\hline Education & 289.2 & 1.3 & 6.0 & 2.2 & 1.7 & 4.1 & 2.9 & 0.7 \\
\hline Health and welfare & 242.6 & 2.7 & 9.8 & 4.5 & 0.8 & 5.4 & 3.8 & 4.8 \\
\hline Private household services & 36.2 & -4.1 & -1.3 & 23.9 & -13.6 & 9.7 & 0.0 & 1.0 \\
\hline Labor input & $\begin{array}{c}\text { In millions } \\
\text { of man-hours } \\
\end{array}$ & & & & & & & \\
\hline Total 3/ & 86.0 & 1.4 & -0.1 & 3.9 & 5.1 & -0.2 & 1.7 & 2.2 \\
\hline Agriculture & 1.8 & -3.7 & -2.6 & 9.8 & -4.6 & -8.2 & -3.4 & 0.5 \\
\hline Industry & 16.0 & -1.2 & -2.8 & 0.5 & 3.9 & -3.1 & -2.2 & 2.8 \\
\hline Public utilities & 0.8 & 0.1 & 6.2 & 0.1 & -0.9 & -3.3 & 2.7 & -8.6 \\
\hline Construction & 5.5 & -3.0 & -13.0 & -7.7 & -0.9 & -2.4 & 4.5 & 7.8 \\
\hline Commerce & 12.4 & 2.0 & 1.6 & 4.1 & 5.0 & -0.3 & 5.5 & -0.5 \\
\hline Hotels and restaurants & 3.5 & -1.7 & 5.2 & 12.2 & 13.9 & -6.2 & -4.9 & 1.0 \\
\hline Transport, storage, and communication & 6.1 & -1.8 & -1.0 & 12.4 & 9.6 & 0.1 & -2.7 & 1.5 \\
\hline Banking, insurance, and finance & 3.0 & 9.2 & -0.8 & 2.6 & -1.6 & 1.4 & 4.8 & 3.3 \\
\hline Business services & 11.4 & 6.2 & 3.4 & 5.3 & 18.7 & 4.2 & -0.7 & 8.7 \\
\hline Community, social, and personal services & 3.7 & 2.5 & -1.5 & 1.0 & 3.4 & 5.4 & 4.1 & -0.2 \\
\hline Civil service & 5.1 & 3.7 & -1.1 & 5.8 & 2.0 & 4.6 & 5.9 & -3.9 \\
\hline Education & 7.2 & 4.1 & 2.4 & 5.0 & -0.6 & 2.1 & 5.3 & 0.2 \\
\hline Health and welfare & 7.7 & 3.3 & 9.1 & 3.3 & 1.7 & 2.2 & 4.1 & 4.2 \\
\hline Private household services & 1.1 & 0.1 & 7.5 & 29.3 & -4.2 & 7.6 & 10.5 & 6.8 \\
\hline
\end{tabular}

Sources: Central Bureau of Statistics, Monthly Bulletin of Statistics; and data provided by the Bank of Israel.

1/ Employment figures are annual averages; labor input figures are weekly averages.

2/ Nine-month figure.

3/ Data do not sum to total due to an "unknown" category. 


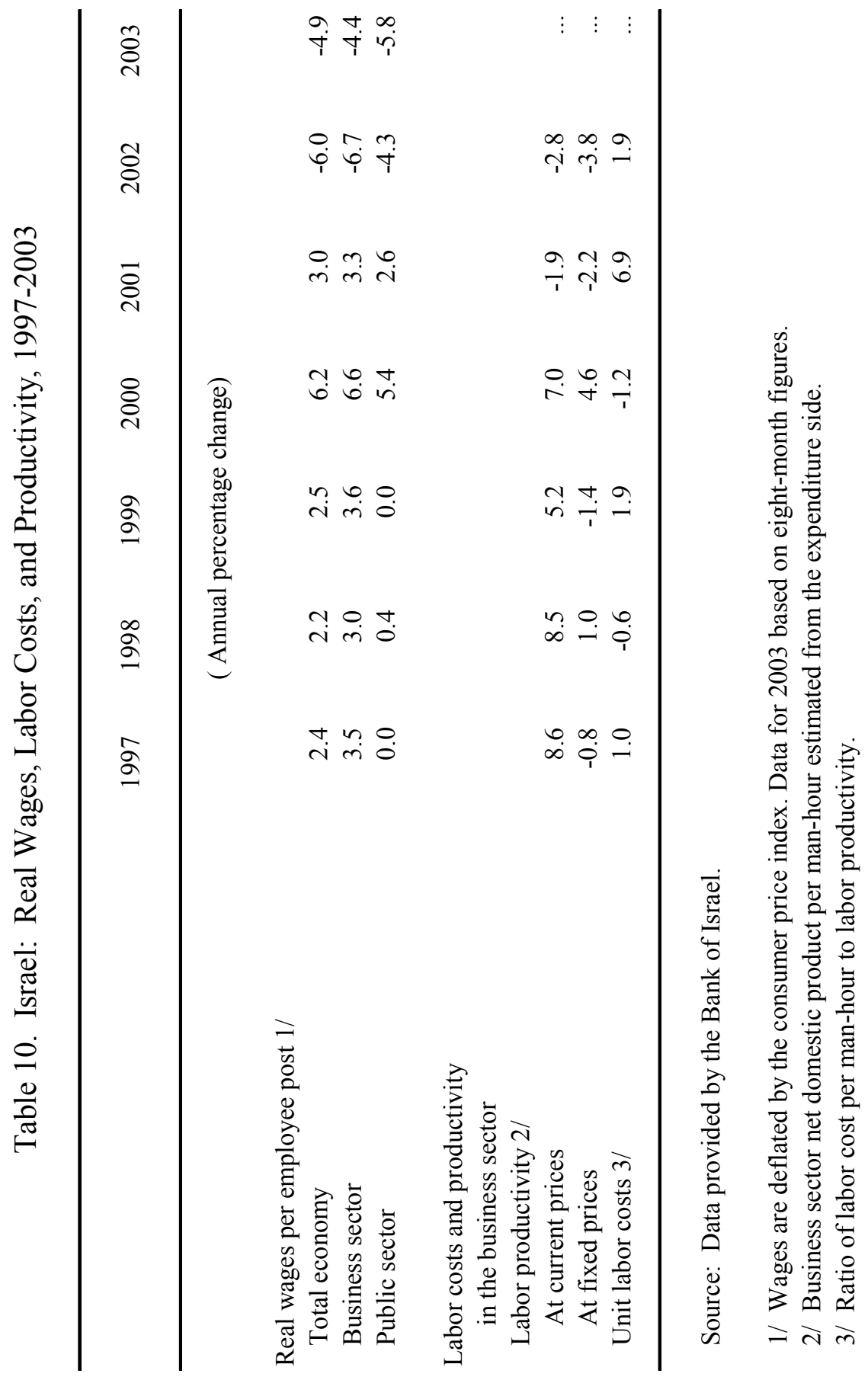


Table 11. Israel: Real Wage Indices, 1997-2003 1/

\begin{tabular}{|c|c|c|c|}
\hline & $\begin{array}{l}\text { Public } \\
\text { Services }\end{array}$ & $\begin{array}{l}\text { Business } \\
\text { Sector }\end{array}$ & Total \\
\hline & & $(2001=100)$ & \\
\hline 1997 & 92.1 & 85.1 & 87.2 \\
\hline 1998 & 92.4 & 87.7 & 89.1 \\
\hline 1999 & 92.5 & 90.8 & 91.4 \\
\hline 2000 & 97.5 & 96.8 & 97.1 \\
\hline 2001 & 100.0 & 100.0 & 100.0 \\
\hline 2002 & 95.7 & 93.3 & 94.0 \\
\hline $20032 /$ & 91.6 & 90.6 & 90.8 \\
\hline \multicolumn{4}{|l|}{1998} \\
\hline I & 90.2 & 88.2 & 88.6 \\
\hline II & 95.6 & 88.0 & 90.0 \\
\hline III & 96.8 & 88.4 & 90.7 \\
\hline IV & 87.2 & 86.0 & 86.2 \\
\hline \multicolumn{4}{|l|}{1999} \\
\hline I & 87.9 & 89.1 & 88.5 \\
\hline II & 95.4 & 90.1 & 91.5 \\
\hline III & 93.5 & 90.7 & 91.4 \\
\hline IV & 93.1 & 93.4 & 93.1 \\
\hline \multicolumn{4}{|l|}{2000} \\
\hline I & 93.8 & 95.7 & 94.8 \\
\hline II & 98.8 & 95.4 & 96.2 \\
\hline III & 102.8 & 96.6 & 98.2 \\
\hline IV & 94.5 & 99.7 & 97.9 \\
\hline \multicolumn{4}{|l|}{2001} \\
\hline I & 96.4 & 101.8 & 99.8 \\
\hline II & 106.0 & 100.5 & 101.9 \\
\hline III & 102.3 & 98.8 & 99.6 \\
\hline IV & 95.3 & 99.0 & 97.5 \\
\hline \multicolumn{4}{|l|}{2002} \\
\hline I & 97.5 & 96.6 & 96.5 \\
\hline II & 98.0 & 95.1 & 95.7 \\
\hline III & 96.3 & 91.0 & 92.3 \\
\hline IV & 90.8 & 90.6 & 90.4 \\
\hline \multicolumn{4}{|l|}{2003} \\
\hline I & 91.1 & 90.5 & 90.3 \\
\hline II & 89.4 & 89.8 & 89.3 \\
\hline July-August & 95.8 & 91.9 & 93.1 \\
\hline
\end{tabular}

Source: Data provided by the Bank of Israel.

$1 /$ Average monthly wage per employee post at constant prices, based on employers' contributions to the National Insurance Institute, deflated by the consumer price index. Data from 1994 are based on a new sample and a new definition of "public services"; data from earlier years were connected via linking indices.

2/ Eight-month figure. 


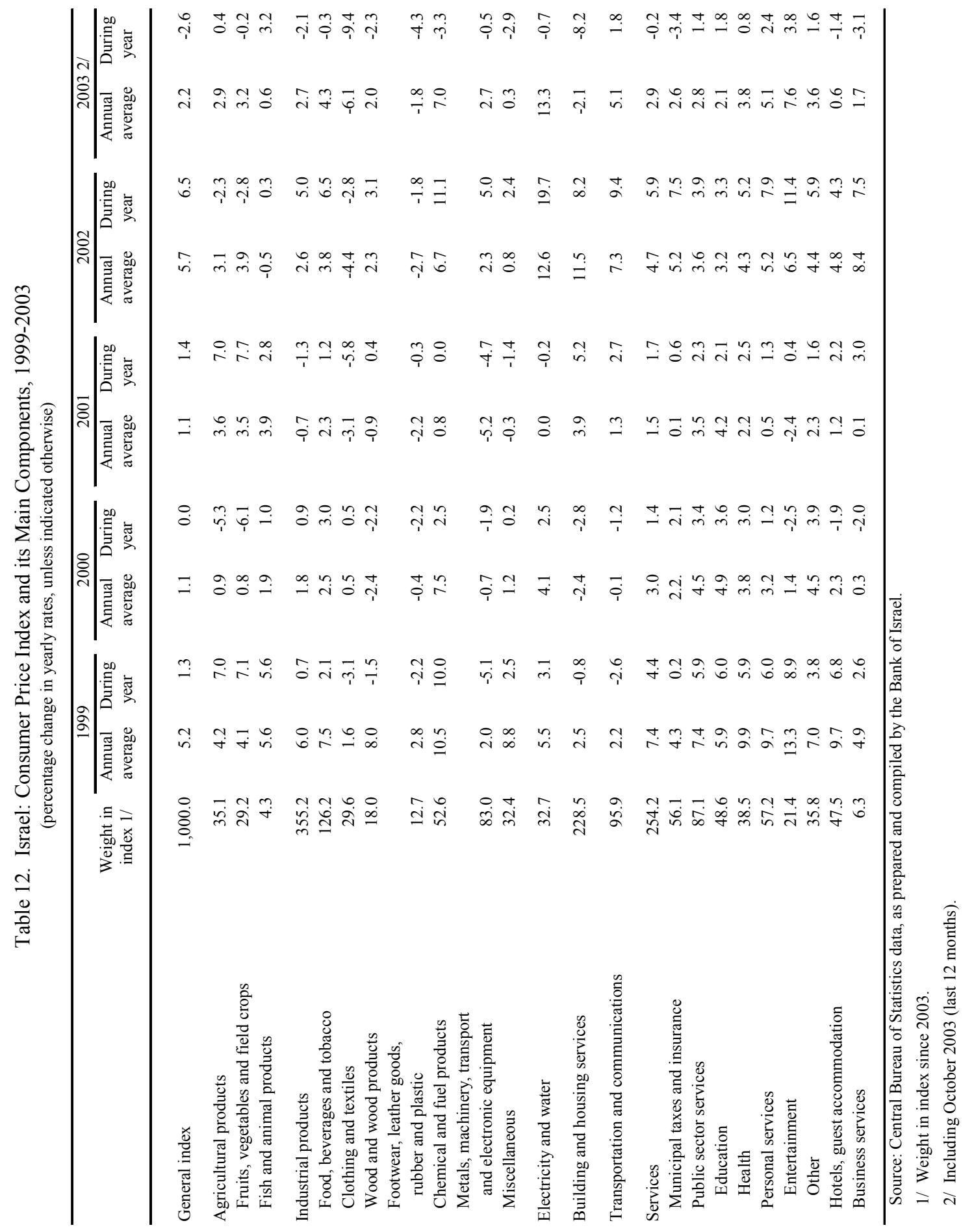


Table 13. Israel: Selected Price Indices, 1997-2002

(Percent increase during the period)

\begin{tabular}{|c|c|c|c|c|c|c|c|c|}
\hline & \multicolumn{2}{|c|}{ Weight $1 /$} & \multirow[b]{2}{*}{1997} & \multirow[b]{2}{*}{1998} & \multirow[b]{2}{*}{1999} & \multirow[b]{2}{*}{2000} & \multirow[b]{2}{*}{2001} & \multirow[b]{2}{*}{2002} \\
\hline & 1999 & 2000 & & & & & & \\
\hline \multicolumn{9}{|l|}{ Consumer price index } \\
\hline General index & $1,000.0$ & $1,000.0$ & 7.0 & 8.6 & 1.3 & 0.0 & 1.4 & 6.5 \\
\hline Controlled prices $2 /$ & 134.5 & 151.0 & 7.6 & 7.6 & 1.2 & 2.2 & -5 & 6.7 \\
\hline Uncontrolled prices & 865.5 & 849.0 & 6.9 & 8.8 & 1.4 & -0.4 & $\ldots$ & $\ldots$ \\
\hline Tradables & 402.2 & 400.8 & 5.8 & 8.7 & -0.1 & -0.4 & -3.9 & 6.6 \\
\hline Nontradables & 597.8 & 599.2 & 7.7 & 8.6 & 2.3 & 0.2 & -5.9 & 6.5 \\
\hline Of which: Housing & 214.2 & 206.4 & 7.5 & 8.8 & -0.9 & -2.4 & $\ldots$ & $\ldots$ \\
\hline CPI excluding housing & 785.8 & 793.0 & 6.8 & 8.6 & 2.0 & 0.6 & 0.4 & 6.1 \\
\hline $\begin{array}{l}\text { CPI excluding housing and } \\
\text { fruits \& vegetables }\end{array}$ & 750.4 & 763.0 & 6.7 & 8.5 & 1.7 & 0.9 & 0.2 & 6.3 \\
\hline $\begin{array}{l}\text { CPI excluding housing, fruits } \& \\
\text { vegetables, controlled prices, } \\
\text { clothing \& footwear }\end{array}$ & 569.9 & 576.5 & 7.8 & 8.8 & 2.4 & 0.6 & -4.2 & 6.9 \\
\hline $\begin{array}{l}\text { Wholesale price index of } \\
\text { industrial output }\end{array}$ & & & 5.9 & 8.2 & 3.5 & 2.0 & -1.9 & 6.9 \\
\hline \multicolumn{9}{|l|}{ Exchange rates } \\
\hline Against the U.S. dollar & & & 7.9 & 18.2 & 0.4 & -2.7 & 4.8 & 9.8 \\
\hline Against the currency basket & & & 3.7 & 20.6 & -2.5 & -6.3 & 3.7 & 14.2 \\
\hline
\end{tabular}

Source: Central Bureau of Statistics data, as prepared and compiled by the Bank of Israel.

1/ The weights of the components in the Consumer Price Index were changed in 2000.

2/ Public transport, communication services, education, medical serivices, municiple taxes, electricity, fuel, water, and me: 
Table 14. Israel: Bank of Israel Accounts, 1997-2002

\begin{tabular}{|c|c|c|c|c|c|c|}
\hline & 1997 & 1998 & 1999 & 2000 & 2001 & 2002 \\
\hline & \multicolumn{6}{|c|}{ (In millions of new sheqalim; end of period) } \\
\hline Total assets & 85,952 & 107,807 & 107,499 & 107,824 & 116,911 & 128,686 \\
\hline Foreign assets & 71,896 & 94,325 & 93,504 & 93,606 & 102,367 & 112,123 \\
\hline International Monetary Fund & $\ldots$ & $\ldots$ & 375 & 474 & 875 & 1,957 \\
\hline Domestic assets & 14,056 & 13,482 & 13,620 & 13,744 & 13,669 & 14,606 \\
\hline Credit to the government (long term debt) & 7,109 & 6,760 & 6,367 & 5,946 & 5,514 & 5,051 \\
\hline Loans and notes & 1,537 & 838 & 810 & 787 & 807 & 3,006 \\
\hline Monetary & 1,444 & 796 & 785 & 781 & 802 & 871 \\
\hline Other & 93 & 42 & 25 & 6 & 5 & 2,135 \\
\hline Government securities & 5,090 & 5,528 & 6,049 & 6,584 & 6,690 & 6,005 \\
\hline Other accounts & 320 & 356 & 394 & 427 & 658 & 544 \\
\hline Total liabilities & 85,952 & 107,807 & 107,499 & 107,824 & 116,911 & 128,686 \\
\hline Foreign liabilities & 617 & 714 & 680 & 633 & 665 & 761 \\
\hline Allocations of SDRs & 511 & 626 & 610 & 564 & 593 & 685 \\
\hline \multicolumn{7}{|l|}{ Liabilities to international monetary } \\
\hline organizations & 106 & 88 & 70 & 69 & 72 & 76 \\
\hline Currency in circulation & 10,373 & 11,935 & 15,605 & 14,659 & 16,858 & 18,009 \\
\hline Deposits of the government & 27,550 & 39,407 & 32,242 & 37,369 & 39,290 & 51,914 \\
\hline In Israeli currency & 14,917 & 18,824 & 12,141 & 16,328 & 20,281 & 31,542 \\
\hline In foreign currency & 12,633 & 20,583 & 20,101 & 21,041 & 19,008 & 20,372 \\
\hline Deposits of banking institutions & 46,547 & 52,709 & 64,461 & 65,900 & 64,216 & 50,266 \\
\hline In Israeli currency & 38,832 & 44,778 & 53,720 & 56,800 & 53,442 & 43,200 \\
\hline In foreign currency & 7,715 & 7,931 & 10,741 & 9,100 & 10,773 & 7,066 \\
\hline Against resident deposits & 7,247 & 7,526 & 8,324 & 8,741 & 9,238 & 4,790 \\
\hline Against nonresident deposits & $\ldots$ & $\ldots$ & $\ldots$ & $\ldots$ & $\ldots$ & $\ldots$ \\
\hline Free deposits of banking institutions & 468 & 405 & 2,417 & 359 & 1,535 & 2,276 \\
\hline Other deposits and accounts & 1,890 & 2,722 & 2,301 & 2,795 & 2,972 & 3,406 \\
\hline Revaluation accounts & $\ldots$ & $\ldots$ & $\ldots$ & 960 & 6,659 & 16,014 \\
\hline Capital and reserves & $-1,025$ & 320 & $-7,790$ & $-14,492$ & $-13,750$ & $-11,684$ \\
\hline
\end{tabular}

Sources: Central Bureau of Statistics, Monthly Bulletin of Statistics; and data provided by the Bank of Israel. 
Table 15. Israel: Financial Assets of the Public,1998-2002

\begin{tabular}{|c|c|c|c|c|c|}
\hline & 1998 & 1999 & 2000 & 2001 & 2002 \\
\hline & \multicolumn{5}{|c|}{ (In millions of new sheqalim; end of period) } \\
\hline Total assets in banks and government bonds & 544,700 & 600,174 & 639,094 & 698,105 & 756,033 \\
\hline Broad money assets & 382,497 & 426,920 & 455,949 & 489,629 & 519,836 \\
\hline Liquid financial assets (M3) & 197,144 & 236,487 & 271,933 & 309,384 & 314,907 \\
\hline Money supply (M1) & 20,591 & 23,536 & 25,298 & 29,198 & 30,628 \\
\hline Time deposits & 101,780 & 130,722 & 160,722 & 184,878 & 182,308 \\
\hline CD'S & 20,153 & 24,544 & 26,365 & 28,782 & 24,835 \\
\hline Special deposits $2 /$ & 1 & 0 & 0 & 0 & 0 \\
\hline Resident foreign currency & 38,547 & 43,239 & 46,766 & 52,666 & 59,967 \\
\hline \multicolumn{6}{|l|}{ Medium-term assets } \\
\hline Restitution deposits & 18,545 & 16,997 & 15,254 & 16,403 & 20,194 \\
\hline \multicolumn{6}{|l|}{ Long-term assets } \\
\hline CPI indexed saving schemes & 95,788 & 101,778 & 93,388 & 91,463 & 108,299 \\
\hline Foreign currency saving schemes & 5,746 & 6,268 & 7,573 & 6,562 & 6,772 \\
\hline Long-term CPI indexed deposits & 58,040 & 58,080 & 60,223 & 60,691 & 63,248 \\
\hline Long-term denominated in and indexed deposits & 7,234 & 7,309 & 7,577 & 5,126 & 6,416 \\
\hline Earmarked deposits & $\ldots$ & $\ldots$ & $\ldots$ & $\ldots$ & $\ldots$ \\
\hline Short-term treasury bills & 15,983 & 18,203 & 25,866 & 26,556 & 37,967 \\
\hline Tradable government bonds & 146,220 & 155,050 & 157,280 & 181,920 & 198,230 \\
\hline Stocks & 190,502 & 191,239 & 191,037 & 263,182 & 201,556 \\
\hline Total financial assets & 735,202 & 791,413 & 830,131 & 961,287 & 957,589 \\
\hline \multirow[t]{2}{*}{ Of which: Nonindexed short-term assets } & 158,509 & 197,005 & 238,251 & 269,414 & 275,737 \\
\hline & \multicolumn{5}{|c|}{ (In percent of total financial assets) } \\
\hline Total assets in banks and government bonds & 74.1 & 75.8 & 77.0 & 72.6 & 79.0 \\
\hline Broad money assets & 52.0 & 53.9 & 54.9 & 50.9 & 54.3 \\
\hline Liquid financial assets (M3) & 26.8 & 29.9 & 32.8 & 32.2 & 32.9 \\
\hline Money supply (M1) & 2.8 & 3.0 & 3.0 & 3.0 & 3.2 \\
\hline Time deposits & 13.8 & 16.5 & 19.4 & 19.2 & 19.0 \\
\hline CD'S & 2.7 & 3.1 & 3.2 & 3.0 & 2.6 \\
\hline Special deposits 2/ & 0.0 & 0.0 & 0.0 & 0.0 & 0.0 \\
\hline Resident foreign currency & 5.2 & 5.5 & 5.6 & 5.5 & 6.3 \\
\hline \multicolumn{6}{|l|}{ Medium-term assets } \\
\hline Restitution deposits & 2.5 & 2.1 & 1.8 & 1.7 & 2.1 \\
\hline \multicolumn{6}{|l|}{ Long-term assets } \\
\hline CPI indexed saving schemes & 13.0 & 12.9 & 11.2 & 9.5 & 11.3 \\
\hline Foreign currency saving schemes & 0.8 & 0.8 & 0.9 & 0.7 & 0.7 \\
\hline Long-term CPI indexed deposits & 7.9 & 7.3 & 7.3 & 6.3 & 6.6 \\
\hline Long-term denominated in and indexed deposits & 1.0 & 0.9 & 0.9 & 0.5 & 0.7 \\
\hline Earmarked deposits & $\ldots$ & $\ldots$ & $\ldots$ & $\ldots$ & $\ldots$ \\
\hline Short-term treasury bills & 2.2 & 2.3 & 3.1 & 2.8 & 4.0 \\
\hline Tradable government bonds & 19.9 & 19.6 & 18.9 & 18.9 & 20.7 \\
\hline Stocks & 25.9 & 24.2 & 23.0 & 27.4 & 21.0 \\
\hline Total financial assets & 100.0 & 100.0 & 100.0 & 100.0 & 100.0 \\
\hline Of which: Nonindexed short-term assets & 21.6 & 24.9 & 28.7 & 28.0 & 28.8 \\
\hline
\end{tabular}


Table 15. Israel: Financial assets of the Public,1998-2002

\begin{tabular}{|c|c|c|c|c|c|}
\hline & 1998 & 1999 & 2000 & 2001 & 2002 \\
\hline & \multicolumn{5}{|c|}{ (Real rate of growth) 3/ } \\
\hline Total assets in banks and government bonds & $\ldots$ & 8.7 & 6.5 & 7.7 & 1.7 \\
\hline Broad money assets & $\ldots$ & 10.1 & 6.8 & 5.9 & -0.3 \\
\hline Liquid financial assets (M3) & $\ldots$ & 18.4 & 15.0 & 12.2 & -4.4 \\
\hline Money supply (M1) & $\ldots$ & 12.8 & 7.5 & 13.8 & -1.5 \\
\hline Time deposits & $\ldots$ & 26.7 & 22.9 & 13.4 & -7.4 \\
\hline CD'S & $\ldots$ & 20.2 & 7.4 & 7.6 & -19.0 \\
\hline Special deposits $2 /$ & $\ldots$ & $\ldots$ & $\ldots$ & $\ldots$ & $\ldots$ \\
\hline Resident foreign currency & $\ldots$ & 10.7 & 8.2 & 11.1 & 6.9 \\
\hline Restitution deposits & $\ldots$ & -9.6 & -10.3 & 6.0 & 15.6 \\
\hline Long-term assets & $\ldots$ & & & & \\
\hline CPI indexed saving schemes & $\ldots$ & 4.8 & -8.2 & -3.4 & 11.2 \\
\hline Foreign currency saving schemes & $\ldots$ & 7.6 & 20.8 & -14.6 & -3.1 \\
\hline Long-term CPI indexed deposits & $\ldots$ & -1.3 & 3.7 & -0.6 & -2.1 \\
\hline Long-term denominated in and indexed deposits & $\ldots$ & -0.3 & 3.7 & -33.3 & 17.5 \\
\hline Earmarked deposits & $\ldots$ & & & & \\
\hline Short-term treasury bills & $\ldots$ & 12.4 & 42.1 & 1.2 & 34.2 \\
\hline Tradable government bonds & $\ldots$ & 4.6 & 1.4 & 14.1 & 2.3 \\
\hline Stocks & $\ldots$ & -0.9 & -0.1 & 35.9 & -28.1 \\
\hline Total financial assets & $\ldots$ & 6.2 & 4.9 & 14.2 & -6.5 \\
\hline Of which: Nonindexed short-term assets & $\ldots$ & 22.6 & 20.9 & 11.5 & -3.9 \\
\hline
\end{tabular}

Source: Data provided by the Bank of Israel.

1/ The public consists of individuals and corporations excluding the Government, Bank of Israel, ordinary banking corporations, and banks abroad. It includes social and life insurance funds but does not include the assets of these funds held out of the ordinary banking system.

2/ Noninterest-bearing deposits mandated by law to cover total subscriptions for securities floatations in the TASE.

3/ Nominal rate deflated by consumer price index. 
Table 16. Israel: Interest Rates, 1997-2004 1/

\begin{tabular}{|c|c|c|c|c|c|c|c|c|c|c|}
\hline & \multirow{2}{*}{$\begin{array}{l}\text { Inflation } \\
\text { rate }\end{array}$} & \multirow{2}{*}{$\begin{array}{l}\text { Change in } \\
\text { NIS/US\$ } \\
\text { exchange } \\
\text { rate }\end{array}$} & \multicolumn{3}{|c|}{ Lending rates } & \multicolumn{3}{|c|}{ Deposit rates } & \multirow{2}{*}{$\begin{array}{c}\text { Yield to } \\
\text { maturity } \\
\text { on treasury } \\
\text { bills }\end{array}$} & \multirow{2}{*}{$\begin{array}{l}\text { Yield to } \\
\text { maturity } \\
\text { on 5-year } \\
\text { bonds }\end{array}$} \\
\hline & & & $\begin{array}{c}\text { Overdraft } \\
\text { facilities 2/ }\end{array}$ & $\begin{array}{c}\text { Exceptional } \\
\text { credit }\end{array}$ & $\begin{array}{c}\text { Nondirected } \\
\text { NIS } \\
\text { credit 3/ }\end{array}$ & $\begin{array}{c}\text { Reserve } \\
\text { deposits } \\
\text { with BoI }\end{array}$ & $\begin{array}{c}\text { Self } \\
\text { Renewing } \\
\text { Overnight }\end{array}$ & $\begin{array}{c}\text { Time } \\
\text { deposits }\end{array}$ & & \\
\hline & \multicolumn{2}{|c|}{ (Average annual rate) } & \multicolumn{8}{|c|}{ (Nominal rates in percent per annum) 4/ } \\
\hline 2000 & 1.1 & -1.5 & 15.8 & 18.1 & 12.8 & 8.0 & 8.0 & 8.6 & 8.8 & 6.0 \\
\hline 2001 & 1.2 & 4.6 & 13.4 & 15.6 & 10.0 & 5.7 & 5.6 & 6.2 & 6.5 & 4.9 \\
\hline 2002 & 5.7 & 15.1 & 13.6 & 15.5 & 9.9 & 5.2 & 5.8 & 6.0 & 7.4 & 4.8 \\
\hline & \multicolumn{2}{|c|}{ (Annualized rate) } & \multicolumn{8}{|c|}{ (Percent increase over preceding month) } \\
\hline 2000 Jan. & 1.3 & -22.5 & 17.3 & 19.4 & 14.6 & 9.4 & 9.4 & 10.0 & 9.4 & 5.9 \\
\hline Feb. & 1.6 & -14.0 & 16.9 & 19.1 & 14.1 & 8.6 & 9.0 & 9.6 & 9.3 & 6.0 \\
\hline Mar. & 1.5 & -13.7 & 16.5 & 18.7 & 13.6 & 8.7 & 8.6 & 9.2 & 8.6 & 5.6 \\
\hline Apr. & 1.7 & 11.4 & 16.1 & 18.3 & 13.2 & 8.3 & 8.2 & 8.9 & 8.8 & 5.7 \\
\hline May & 2.1 & 36.2 & 15.9 & 18.1 & 12.9 & 8.0 & 8.0 & 8.6 & 9.1 & 5.7 \\
\hline June & 2.1 & -10.6 & 15.9 & 18.0 & 12.9 & 7.9 & 8.0 & 8.6 & 9.3 & 5.7 \\
\hline July & 2.1 & -5.0 & 15.8 & 18.1 & 12.9 & 8.0 & 7.9 & 8.6 & 9.0 & 6.1 \\
\hline Aug. & 1.0 & -11.5 & 15.6 & 17.9 & 12.6 & 7.8 & 7.8 & 8.4 & 8.8 & 6.5 \\
\hline Sep. & 0.0 & -2.8 & 15.2 & 17.7 & 12.3 & 7.6 & 7.5 & 8.2 & 8.6 & 6.0 \\
\hline Oct. & -0.1 & 21.7 & 15.1 & 17.4 & 12.0 & 7.4 & 7.2 & 7.9 & 8.6 & 6.1 \\
\hline Nov. & 0.1 & 1.3 & 15.0 & 17.3 & 11.8 & 7.4 & 7.1 & 7.8 & 8.4 & 6.1 \\
\hline Dec. & 0.0 & -8.0 & 14.6 & 17.0 & 11.5 & 7.0 & 6.9 & 7.6 & 7.8 & 6.2 \\
\hline 2001 Jan. & -0.1 & 11.8 & 14.6 & 16.8 & 11.3 & 6.9 & 6.7 & 7.4 & 7.6 & 6.1 \\
\hline Feb. & 0.3 & 0.6 & 14.2 & 16.5 & 11.0 & 6.6 & 6.5 & 7.1 & 7.2 & 5.7 \\
\hline Mar. & 0.7 & 13.3 & 14.0 & 16.3 & 10.8 & 6.4 & 6.3 & 6.88 & 7.1 & 5.4 \\
\hline Apr. & 1.2 & 3.3 & 13.7 & 16.0 & 10.4 & 6.1 & 5.9 & 6.61 & 6.8 & 5.2 \\
\hline May & $\ldots$ & -9.9 & 13.7 & $\ldots$ & 10.4 & $\ldots$ & 6.0 & 6.52 & 6.6 & 5.0 \\
\hline June & $\ldots$ & 2.6 & 13.4 & $\ldots$ & 10.1 & $\ldots$ & 5.7 & 6.28 & 6.3 & 4.7 \\
\hline July & $\ldots$ & $\ldots$ & 13.1 & $\ldots$ & 9.7 & $\ldots$ & 5.3 & 5.9 & 6.2 & 4.5 \\
\hline Aug. & 1.7 & 10.5 & 12.8 & 14.9 & 9.4 & 5.2 & 5.1 & 5.7 & 6.4 & 4.3 \\
\hline Sep. & 2.5 & 28.4 & 13.0 & 15.2 & 9.4 & 5.1 & 5.1 & 5.7 & 6.7 & 4.1 \\
\hline Oct. & 2.0 & -2.1 & 13.2 & 15.3 & 9.5 & 5.2 & 5.1 & 5.7 & 6.3 & 4.4 \\
\hline Nov. & 1.4 & -19.2 & 12.8 & 14.9 & 9.3 & 5.0 & 4.9 & 5.5 & 5.8 & 4.7 \\
\hline Dec. & 1.4 & 10.9 & 12.0 & 14.3 & 8.6 & 4.4 & 4.2 & 4.9 & 5.0 & 4.4 \\
\hline $2002 \mathrm{Jan}$ & 3.1 & 103.8 & 10.4 & 12.6 & 7.2 & 3.1 & 2.9 & 3.6 & 4.3 & 3.1 \\
\hline Feb. & 4.0 & 38.5 & 10.5 & 12.6 & 7.2 & 3.0 & 2.9 & 3.5 & 4.8 & 2.9 \\
\hline Mar. & 4.3 & 0.6 & 11.2 & 13.4 & 7.7 & 3.3 & 3.4 & 3.8 & 5.3 & 3.8 \\
\hline Apr. & 5.0 & 43.3 & 11.2 & 13.2 & 7.7 & 3.3 & 3.4 & 3.9 & 6.0 & 4.3 \\
\hline May & 5.5 & 24.3 & 11.6 & 13.6 & 7.8 & 3.4 & 3.7 & 4.0 & 6.7 & 4.2 \\
\hline June & 6.6 & 11.7 & 13.7 & 15.6 & 9.7 & 4.9 & 5.7 & 5.6 & 8.9 & 4.6 \\
\hline July & 6.9 & -41.9 & 16.1 & 17.8 & 11.8 & 6.9 & 8.0 & 7.8 & 8.9 & 5.7 \\
\hline Aug. & 6.2 & -10.8 & 16.1 & 17.8 & 11.9 & 6.9 & 7.9 & 7.9 & 8.7 & 5.7 \\
\hline Sep. & 6.4 & 28.5 & 15.9 & 17.8 & 12.0 & 7.0 & 7.9 & 8.0 & 8.9 & 5.9 \\
\hline Oct. & 6.9 & 6.0 & 15.5 & 17.2 & 11.8 & 7.0 & 7.9 & 8.0 & 9.3 & 6.0 \\
\hline Nov. & 6.7 & -24.6 & 15.5 & 17.2 & 11.8 & 6.9 & 7.9 & 8.1 & 8.9 & 5.9 \\
\hline Dec. & 6.5 & 1.8 & 15.3 & 17.2 & 11.8 & 7.0 & 7.7 & 8.1 & 7.9 & 5.8 \\
\hline $2003 \mathrm{Jan}$ & 5.6 & 43.2 & 15.8 & 16.8 & 12.0 & 6.6 & 7.7 & 8.0 & 8.1 & 5.7 \\
\hline Feb. & 5.1 & 7.6 & 15.7 & 17.0 & 12.1 & 6.7 & 7.7 & 8.0 & 8.8 & 5.5 \\
\hline Mar. & 4.8 & -19.1 & 15.2 & 16.7 & 11.9 & 6.5 & 7.7 & 8.0 & 8.6 & 5.5 \\
\hline Apr. & 3.1 & -34.0 & 15.2 & 16.5 & 11.8 & 6.3 & 7.5 & 7.8 & 8.1 & 5.7 \\
\hline May & 1.6 & -31.0 & 14.7 & 16.2 & 11.5 & 6.1 & 7.2 & 7.5 & 7.6 & 5.3 \\
\hline June & -0.3 & -23.7 & 14.0 & 15.8 & 11.0 & 5.6 & 6.6 & 7.1 & 7.1 & 4.8 \\
\hline July & -1.6 & -1.9 & 13.4 & 15.3 & 10.7 & 5.3 & 6.2 & 6.7 & 6.7 & 4.4 \\
\hline Aug. & -1.1 & 24.7 & 13.0 & 15.0 & 10.2 & 4.7 & 5.7 & 6.2 & 6.6 & 4.6 \\
\hline Sep. & -1.9 & 3.5 & 12.4 & 14.5 & 9.8 & 4.5 & 5.2 & 5.7 & 6.2 & 4.6 \\
\hline Oct. & -2.6 & -4.4 & 12.2 & 14.2 & 9.4 & 4.4 & 4.9 & 5.4 & 5.8 & 4.4 \\
\hline Nov. & -2.0 & 13.0 & 11.7 & 13.8 & 8.9 & 3.8 & 4.3 & 4.9 & 5.4 & 4.2 \\
\hline Dec. & -1.9 & -23.8 & $\ldots$ & $\ldots$ & $\ldots$ & $\ldots$ & $\ldots$ & $\ldots$ & 4.9 & 4.1 \\
\hline 2004 Jan. & $\ldots$ & 8.7 & $\ldots$ & $\ldots$ & $\ldots$ & $\ldots$ & $\ldots$ & $\ldots$ & 4.7 & 3.8 \\
\hline Feb. & $\ldots$ & 14.7 & $\ldots$ & $\ldots$ & $\ldots$ & $\ldots$ & $\ldots$ & $\ldots$ & 4.8 & 3.7 \\
\hline
\end{tabular}

Source: Data provided by the Bank of Israel.

1/ All interest rates converted to annual terms at compounded interest.

2 / Includes basic interest rate, commitment fees and the higher interest charged for drawings in excess of approved ceiling.

3 / Includes interest on overdraft facilities.

4/ Monthly rate compounded annually. 


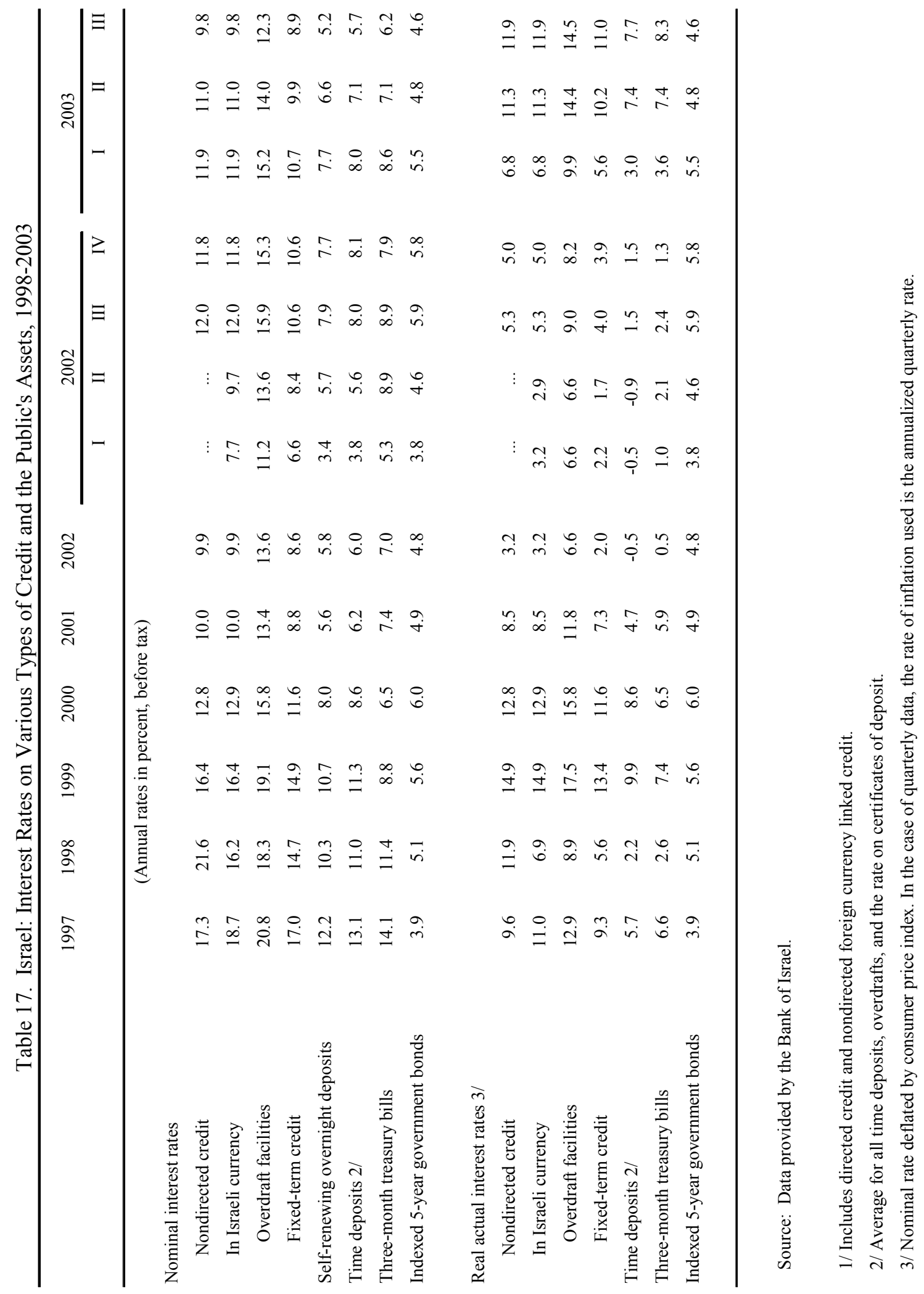


Table 18. Israel: Reserve Requirements on Deposits and Interest Brackets, 1994-2003

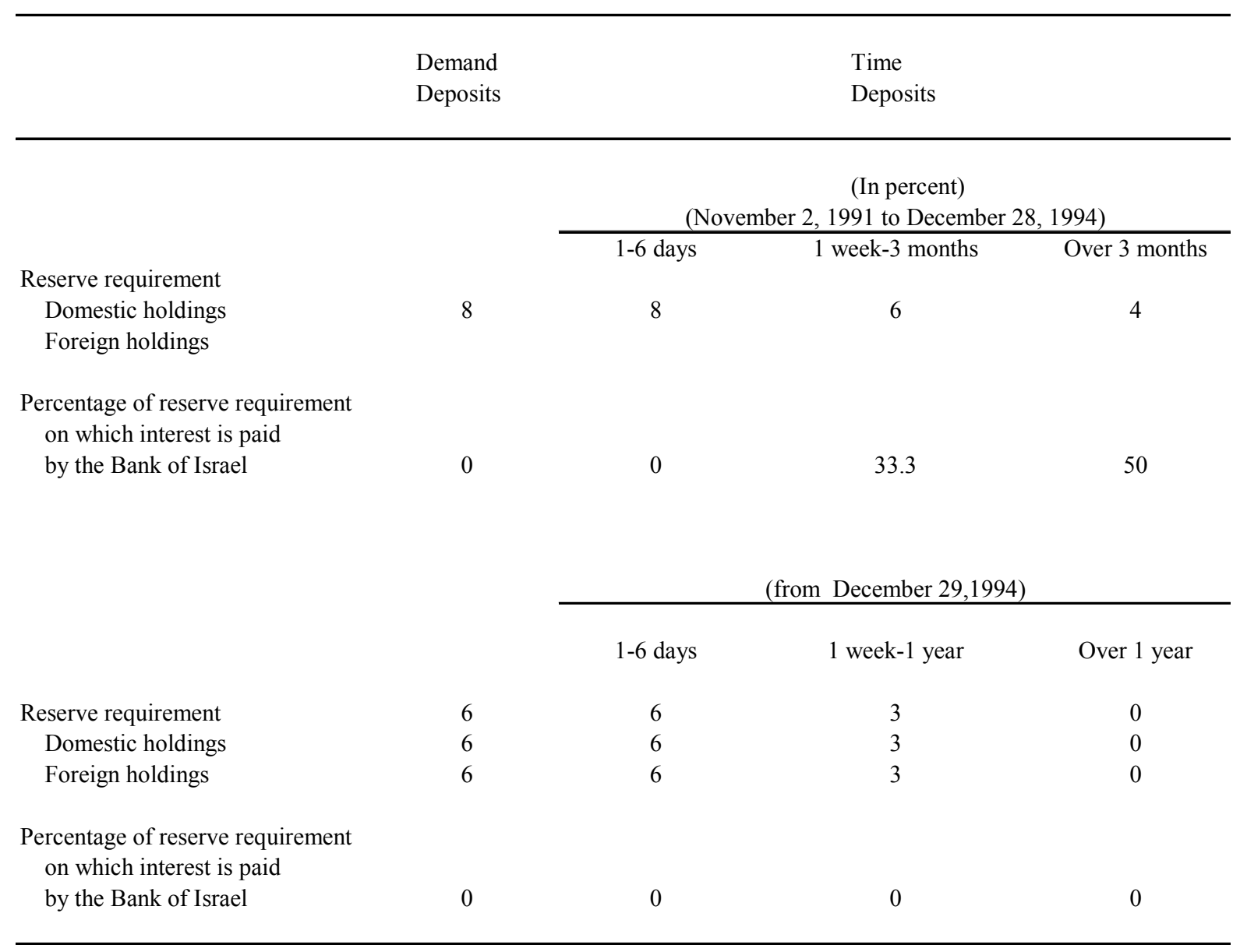

Source: Bank of Israel. 
Table 19. The Israeli Capital Market, 2002

\begin{tabular}{|c|c|c|c|c|c|c|}
\hline & Total & Shares 1/ & $\begin{array}{l}\text { Tradable } \\
\text { Bonds 2/ }\end{array}$ & $\begin{array}{l}\text { Indexed } \\
\text { Earmarked } \\
\text { Bonds }\end{array}$ & $\begin{array}{c}\text { Treasury Bills } \\
\text { 2/ }\end{array}$ & $\begin{array}{c}\text { Other Assets } \\
\text { Held by } \\
\text { Institutions }\end{array}$ \\
\hline & & (In b & llions of new & sheqalim; en & d of period ) & \\
\hline \multicolumn{7}{|l|}{ Institutions } \\
\hline Provident funds & 167.6 & 19.5 & 80.4 & 9.4 & 1.7 & 56.6 \\
\hline Old pension funds & 121.8 & $\ldots$ & 0.0 & 116.1 & $\ldots$ & 5.4 \\
\hline New pension funds & 14.0 & $\ldots$ & 2.4 & 10.0 & $\ldots$ & 1.6 \\
\hline Life insurance & 86.8 & 5.2 & 23.1 & 31.9 & $\ldots$ & 26.5 \\
\hline Mutual funds 3/ & 46.6 & 4.7 & 21.7 & $\ldots$ & 9.7 & 10.5 \\
\hline Households and firms & 138.2 & 78.4 & 38.6 & $\ldots$ & 21.2 & $\ldots$ \\
\hline Nonresidents & 49.6 & 49.2 & 0.2 & $\ldots$ & $\ldots$ & $\ldots$ \\
\hline Banks & 47.7 & $\ldots$ & 36.1 & $\ldots$ & 11.7 & $\ldots$ \\
\hline \multirow[t]{2}{*}{ Total } & 672.3 & 157.2 & 202.5 & 167.5 & 44.4 & 100.7 \\
\hline & \multicolumn{6}{|c|}{ Real change (in percent) } \\
\hline \multicolumn{7}{|l|}{ Institutions } \\
\hline Provident funds & -8.7 & -25.5 & -6.0 & -2.3 & -1.8 & -6.4 \\
\hline Old pension funds & 4.3 & $\ldots$ & -35.2 & 2.6 & $\ldots$ & 67.8 \\
\hline New pension funds & 23.7 & $\ldots$ & 8.4 & 33.2 & $\ldots$ & 0.0 \\
\hline Life insurance & 3.6 & -14.3 & 37.2 & 5.1 & $\ldots$ & -13.0 \\
\hline Mutual funds 3/ & -30.6 & -40.3 & -27.7 & $\ldots$ & -40.7 & -18.5 \\
\hline Households and firms & -5.4 & -23.7 & 16.1 & $\ldots$ & 111.2 & $\ldots$ \\
\hline Non-residents & -12.4 & -12.8 & 117.3 & $\ldots$ & $\ldots$ & $\ldots$ \\
\hline Banks & -12.4 & -12.8 & 117.3 & $\ldots$ & $\cdots$ & $\cdots$ \\
\hline Total & -5.0 & -21.2 & 1.0 & 4.2 & 16.7 & -7.4 \\
\hline $\begin{array}{l}\text { Real change in price } \\
\text { (in percent) }\end{array}$ & -7.3 & -16.2 & -8.8 & -0.1 & $\ldots$ & -0.1 \\
\hline $\begin{array}{l}\text { Real change in quantity } \\
\text { (in percent) }\end{array}$ & 2.5 & -6.0 & 10.8 & 4.3 & 16.7 & -7.3 \\
\hline
\end{tabular}

Source: Bank of Israel, Research Department.

1/ Excluding double counting and government-owned quoted companies.

2/ Excluding securities held by the Bank of Israel.

3/ Adjusted for provident fund and non-residents' holdings in mutual funds. 
Table 20. Israel: Prinicipal Stock Market Indicators, 1997-2002

\begin{tabular}{|c|c|c|c|c|c|c|}
\hline & 1997 & 1998 & 1999 & 2000 & 2001 & 2002 \\
\hline & \multicolumn{6}{|c|}{ (In millions of constant 1996 new sheqalim; unless otherwise indicated) } \\
\hline $\begin{array}{l}\text { Private sector stock offering } \\
\text { excluding privitizations } 1 / 2 \text { / }\end{array}$ & 5,932 & 5,690 & 2,303 & 5,100 & 2,612 & 1,549 \\
\hline Market value 2/ & 153,177 & 146,430 & 230,503 & 229,099 & 220,357 & 158,466 \\
\hline $\begin{array}{l}\text { Volume of trade on stock } \\
\text { exchange } 2 /\end{array}$ & 46,134 & 48,889 & 74,105 & 98,177 & 54,107 & 47,291 \\
\hline Annual turnover ratio 3 / & 0.32 & 0.33 & 0.40 & 0.40 & 0.29 & 0.27 \\
\hline $\begin{array}{l}\text { Real overal rate of return } \\
\text { on all shares }\end{array}$ & $27.2 \%$ & $-5.3 \%$ & $63.5 \%$ & $0.5 \%$ & $-8.2 \%$ & $-25.0 \%$ \\
\hline
\end{tabular}

Sources: Bank of Israel; and Central Bureau of Statistics.

1/ Shares, convertible securities, and exercised options.

2/ Market value end-of-year figures; volume of trade-on and off.

3/ Ratio of monthly volume of trade to average monthly market value of stock market. 
Table 21. Israel: Principal Bond Market Indicators, 1997-2002

\begin{tabular}{|c|c|c|c|c|c|c|}
\hline & 1997 & 1998 & 1999 & 2000 & 2001 & 2002 \\
\hline & \multicolumn{6}{|c|}{$\begin{array}{l}\text { (In millions of constant } 1996 \text { new sheqalim; } \\
\text { unless otherwise indicated) }\end{array}$} \\
\hline \multicolumn{7}{|l|}{ Market value of listed bonds } \\
\hline Government & 125,977 & 125,821 & 131,650 & 133,544 & 146,390 & 155,851 \\
\hline Corporate & 9,328 & 9,706 & 9,858 & 8,627 & 9,986 & 11,699 \\
\hline Total & 135,305 & 135,528 & 141,508 & 142,171 & 156,376 & 167,549 \\
\hline \multicolumn{7}{|l|}{ Of which: } \\
\hline \multicolumn{7}{|l|}{ Percentage held by: } \\
\hline Public & 26.77 & 23.98 & 23.38 & 17.43 & 27.76 & 29.75 \\
\hline Commerical banks & 23.41 & 24.40 & 23.24 & 27.19 & 17.38 & 18.57 \\
\hline Provident and Pension Fund & 41.46 & 41.67 & 42.38 & 43.23 & 42.47 & 39.68 \\
\hline Bank of Israel & 3.54 & 3.50 & 3.62 & 3.92 & 3.51 & 2.82 \\
\hline \multicolumn{7}{|l|}{ Volume of stock exchange trade } \\
\hline Government & 22,339 & 31,625 & 40,805 & 46,439 & 77,215 & 126,822 \\
\hline Corporate bonds $1 /$ & 730 & 752 & 751 & 667 & 867 & 1,490 \\
\hline Total & 23,069 & 32,378 & 41,557 & 47,106 & 78,082 & 128,313 \\
\hline $\begin{array}{l}\text { Bank of Israel operations } 2 / \\
\text { (in percent) }\end{array}$ & 1.34 & 1.06 & 1.25 & 1.13 & 0.45 & 0.07 \\
\hline \multicolumn{7}{|l|}{$\begin{array}{l}\text { Net issues of tradable bonds } \\
\text { (issues less redemptions) }\end{array}$} \\
\hline Government & $-5,173$ & $-1,973$ & $-1,603$ & $-7,183$ & 2,175 & 1,588 \\
\hline Corporate $1 /$ & $-2,330$ & $-1,001$ & -118 & -770 & 1,416 & 1,009 \\
\hline Total & $-7,503$ & $-2,974$ & $-1,721$ & $-7,953$ & 3,591 & 2,598 \\
\hline \multicolumn{7}{|l|}{ Annual turnover 3/ } \\
\hline Government & 0.18 & 0.27 & 0.32 & 0.35 & 0.61 & 0.85 \\
\hline Corporate $1 /$ & 0.08 & 0.09 & 0.08 & 0.07 & 0.10 & 0.14 \\
\hline Total & 0.17 & 0.25 & 0.31 & 0.33 & 0.57 & 0.80 \\
\hline \multicolumn{7}{|l|}{ Real overall rate of return 4/ } \\
\hline CPI-indexed bonds & $4.1 \%$ & $-1.2 \%$ & $3.4 \%$ & $2.1 \%$ & $13.5 \%$ & $-5.3 \%$ \\
\hline Exchange-rate indexed bonds & $6.1 \%$ & $12.0 \%$ & $2.6 \%$ & $3.0 \%$ & $11.0 \%$ & $1.5 \%$ \\
\hline Nominal bonds & $8.8 \%$ & $2.7 \%$ & $12.2 \%$ & $12.0 \%$ & $8.5 \%$ & $-8.3 \%$ \\
\hline
\end{tabular}

Sources: Bank of Israel; and Central Bureau of Statistics.

1/ Including public sector corporations.

2/ Ratio of the central bank's sales and purchases in the secondary market to total volume of stock exchange trade in bonds.

3/ Ratio of monthly volume of trade to average monthly market value of bonds.

4/ Real overall rate of return refers to total return (including coupon), less taxable profits. 
Table 22. Israel: Institutional Investor Indicators, 1997-2002

\begin{tabular}{|c|c|c|c|c|c|c|}
\hline & 1997 & 1998 & 1999 & 2000 & 2001 & 2002 \\
\hline \multicolumn{7}{|l|}{ Mutual funds } \\
\hline Total assets (in NIS millions) & 20,358 & 22,893 & 35,917 & 45,833 & 63,012 & 44,084 \\
\hline Growth rate of total assets (in percent) & 54.4 & 12.5 & 56.9 & 27.6 & 37.5 & -30.0 \\
\hline Real annual rate of return (in percent) & 15.6 & 1.4 & 23.5 & 4.4 & $\ldots$ & $\ldots$ \\
\hline \multicolumn{7}{|l|}{ Percent of total tradable assets: } \\
\hline Bonds & 0.1 & 0.1 & 0.1 & 0.1 & $\ldots$ & $\ldots$ \\
\hline Stocks & 0.1 & 0.0 & 0.0 & 0.0 & $\ldots$ & ... \\
\hline \multicolumn{7}{|l|}{ Provident funds } \\
\hline Total assets (in NIS billions) & 120.0 & 120.0 & 132.0 & 136.0 & $\ldots$ & ... \\
\hline Growth rate of total assets (in percent) & 9.1 & 0.0 & 10.0 & 3.0 & $\ldots$ & $\ldots$ \\
\hline Real annual rate of return (in percent) & 6.7 & 1.2 & 8.6 & 3.0 & $\ldots$ & $\ldots$ \\
\hline \multicolumn{7}{|l|}{ Percent of total tradable assets: } \\
\hline Tradable government bonds & 39.7 & 39.6 & 40.0 & 41.1 & $\ldots$ & $\ldots$ \\
\hline Corporate bonds & 61.3 & 62.5 & 63.4 & 54.4 & $\ldots$ & $\ldots$ \\
\hline Stock & 9.7 & 10.1 & 9.2 & 9.5 & $\ldots$ & $\ldots$ \\
\hline Treasury bills & 24.3 & 16.8 & 12.0 & 17.1 & $\ldots$ & .. \\
\hline Pension funds' assets (in NIS billions) & 84 & 85 & 91 & 95 & $\ldots$ & $\ldots$ \\
\hline
\end{tabular}

Sources: Data provided by the Bank of Israel; and Central Bureau of Statistics. 
Table 23. Israel: State Budget Balance and Financing, 1999-2003

\begin{tabular}{|c|c|c|c|c|c|c|c|c|}
\hline & $\begin{array}{r}1999 \\
\text { Budget }\end{array}$ & $\begin{array}{r}1999 \\
\text { Outturn }\end{array}$ & $\begin{array}{r}2000 \\
\text { Budget }\end{array}$ & $\begin{array}{r}2000 \\
\text { Outturn }\end{array}$ & $\begin{array}{r}2001 \\
\text { Budget }\end{array}$ & $\begin{array}{r}2001 \\
\text { Outturn }\end{array}$ & $\begin{array}{r}2002 \\
\text { Budget }\end{array}$ & $\begin{array}{r}2003 \\
\text { Budget }\end{array}$ \\
\hline & \multicolumn{8}{|c|}{ (In millions of new sheqalim) } \\
\hline Total revenue & 158,248 & 153,496 & 165,757 & 172,393 & 181,135 & 167,958 & 185,497 & 192,239 \\
\hline Total expenditure & 178,056 & 175,012 & 188,927 & 187,218 & 201,001 & 198,715 & 212,428 & 220,903 \\
\hline Budget balance before foreign grants & $-19,808$ & $-21,516$ & $-23,170$ & $-14,825$ & $-19,866$ & $-30,757$ & $-26,931$ & $-28,664$ \\
\hline Foreign grants & 11,808 & 11,049 & 12,260 & 11,588 & 11,466 & 9,755 & 12,281 & 13,464 \\
\hline Budget balance after foreign grants & $-8,000$ & $-10,467$ & $-10,910$ & $-3,237$ & $-8,400$ & $-21,002$ & $-14,650$ & $-15,200$ \\
\hline Total financing & 8,000 & 10,467 & 10,910 & 3,237 & 8,400 & 21,002 & 14,650 & 15,200 \\
\hline Foreign (net) & 884 & -281 & 1,695 & -751 & 0 & $-2,918$ & $-1,000$ & 0 \\
\hline Gross & 9,676 & 6,728 & 10,076 & 5,707 & 10,259 & 5,950 & 9,287 & 16,859 \\
\hline Repayment & 8,792 & 7,008 & 8,381 & 6,457 & 10,259 & 8,868 & 10,287 & 16,859 \\
\hline Domestic (net) & 7,116 & 11,039 & 9,215 & 4,318 & 8,400 & 17,436 & 15,650 & 15,200 \\
\hline Nonbank borrowing (net) & 2,623 & 7,463 & 5,244 & -409 & 3,302 & 16,762 & 13,361 & 13,837 \\
\hline Gross & 32,495 & 38,442 & 38,442 & 31,591 & 44,335 & 52,717 & 47,567 & 52,150 \\
\hline Repayment & 29,872 & 30,979 & 33,198 & 32,000 & 41,033 & 35,954 & 34,206 & 38,313 \\
\hline Bank of Israel credit (net) & 0 & -291 & 0 & -330 & 0 & 6,484 & 0 & 0 \\
\hline Sale of assets & 4,590 & 2,122 & 3,500 & 2,889 & 3,000 & 172 & 1,500 & 1,500 \\
\hline Lending (net) (-) & -97 & 1,455 & 471 & 1,837 & 2,098 & 501 & 789 & -137 \\
\hline Gross (-) & $-5,244$ & $-3,715$ & $-5,249$ & $-3,657$ & $-4,586$ & $-3,636$ & $-5,002$ & $-5,074$ \\
\hline Of which: housing (-) & $-4,194$ & $-3,153$ & $-4,346$ & $-2,940$ & $-3,708$ & $-3,136$ & $-3,901$ & $-4,115$ \\
\hline Repayment & 5,147 & 5,170 & 5,720 & 5,495 & 6,684 & 4,137 & 5,791 & 4,937 \\
\hline Residual & $\ldots$ & $\ldots$ & $\ldots$ & $\ldots$ & $\ldots$ & $\ldots$ & $\ldots$ & $\ldots$ \\
\hline \multicolumn{9}{|l|}{ Memorandum items: } \\
\hline Primary balance & 12,296 & 11,662 & 11,939 & 21,553 & 17,458 & 3,990 & 11,547 & 15,441 \\
\hline Current balance & 8,581 & $-3,322$ & 3,249 & 4,533 & 6,561 & $-12,733$ & -737 & -27 \\
\hline Domestic balance & $-5,985$ & $-8,023$ & $-7,494$ & $-2,505$ & $-2,507$ & $-16,918$ & $-12,984$ & $-11,558$ \\
\hline \multirow[t]{2}{*}{ Foreign balance } & $-2,015$ & $-2,444$ & $-3,416$ & -732 & $-5,893$ & $-4,084$ & $-1,666$ & $-3,642$ \\
\hline & \multicolumn{8}{|c|}{ (In percent of GDP) } \\
\hline Total revenue & 37.1 & 36.0 & 35.7 & 37.1 & 38.5 & 35.7 & 38.4 & 39.8 \\
\hline Total expenditure & 41.7 & 41.0 & 40.7 & 40.3 & 42.8 & 42.3 & 44.0 & 45.7 \\
\hline Budget balance before foreign grants & -4.6 & -5.0 & -5.0 & -3.2 & -4.2 & -6.5 & -5.6 & -5.9 \\
\hline Foreign grants & 2.8 & 2.6 & 2.6 & 2.5 & 2.4 & 2.1 & 2.5 & 2.8 \\
\hline Budget balance after foreign grants & -1.9 & -2.5 & -2.3 & -0.7 & -1.8 & -4.5 & -3.0 & -3.1 \\
\hline Total financing & 1.9 & 2.5 & 2.3 & 0.7 & 1.8 & 4.5 & 3.0 & 3.1 \\
\hline Foreign (net) & 0.2 & -0.1 & 0.4 & -0.2 & 0.0 & -0.6 & -0.2 & 0.0 \\
\hline Gross & 2.3 & 1.6 & 2.2 & 1.2 & 2.2 & 1.3 & 1.9 & 3.5 \\
\hline Repayment & 2.1 & 1.6 & 1.8 & 1.4 & 2.2 & 1.9 & 2.1 & 3.5 \\
\hline Domestic (net) & 1.7 & 2.6 & 2.0 & 0.9 & 1.8 & 3.7 & 3.2 & 3.1 \\
\hline Nonbank borrowing (net) & 0.6 & 1.7 & 1.1 & -0.1 & 0.7 & 3.6 & 2.8 & 2.9 \\
\hline Gross & 7.6 & 9.0 & 8.3 & 6.8 & 9.4 & 11.2 & 9.8 & 10.8 \\
\hline Repayment & 7.0 & 7.3 & 7.1 & 6.9 & 8.7 & 7.6 & 7.1 & 7.9 \\
\hline Bank of Israel credit (net) & 0.0 & -0.1 & 0.0 & -0.1 & 0.0 & 1.4 & 0.0 & 0.0 \\
\hline Sale of assets & 1.1 & 0.5 & 0.8 & 0.6 & 0.6 & 0.0 & 0.3 & 0.3 \\
\hline Lending (net) (-) & 0.0 & 0.3 & 0.1 & 0.4 & 0.4 & 0.1 & 0.2 & 0.0 \\
\hline Gross (-) & -1.2 & -0.9 & -1.1 & -0.8 & -1.0 & -0.8 & -1.0 & -1.0 \\
\hline Of which: housing (-) & -1.0 & -0.7 & -0.9 & -0.6 & -0.8 & -0.7 & -0.8 & -0.9 \\
\hline Repayment & 1.2 & 1.2 & 1.2 & 1.2 & 1.4 & 0.9 & 1.2 & 1.0 \\
\hline \multicolumn{9}{|l|}{ Memorandum items: } \\
\hline Primary balance & 2.9 & 2.7 & 2.6 & 4.6 & 3.7 & 0.8 & 2.4 & 3.2 \\
\hline Current balance & 2.0 & -0.8 & 0.7 & 1.0 & 1.4 & -2.7 & -0.2 & 0.0 \\
\hline Domestic balance & -1.4 & -1.9 & -1.6 & -0.5 & -0.5 & -3.6 & -2.7 & -2.4 \\
\hline Foreign balance & -0.5 & -0.6 & -0.7 & -0.2 & -1.3 & -0.9 & -0.3 & -0.8 \\
\hline
\end{tabular}

Source: Data provided by the Ministry of Finance. 
Table 24. Israel: State Budget Revenue, 1999-2003

\begin{tabular}{|c|c|c|c|c|c|c|c|c|}
\hline & $\begin{array}{r}1999 \\
\text { Budget }\end{array}$ & $\begin{array}{r}1999 \\
\text { Outturn }\end{array}$ & $\begin{array}{r}2000 \\
\text { Budget }\end{array}$ & $\begin{array}{r}2000 \\
\text { Outturn }\end{array}$ & $\begin{array}{r}2001 \\
\text { Budget }\end{array}$ & $\begin{array}{r}2001 \\
\text { Outturn }\end{array}$ & $\begin{array}{r}2002 \\
\text { Budget }\end{array}$ & $\begin{array}{c}2003 \\
\text { Budget }\end{array}$ \\
\hline & \multicolumn{8}{|c|}{ (In millions of new sheqalim) } \\
\hline Total tax revenue & 126,450 & 125,008 & 132,850 & 143,635 & 149,620 & 143,794 & 151,030 & 155,186 \\
\hline Taxes on income and profits & 60,731 & 60,471 & 62,160 & 75,589 & 73,300 & 76,111 & 76,250 & 72,720 \\
\hline Companies & 13,100 & 13,724 & 14,000 & 22,536 & 19,100 & 19,138 & 18,500 & 17,240 \\
\hline Individuals & 47,631 & 46,748 & 48,160 & 53,054 & 54,200 & 56,973 & 57,750 & 55,480 \\
\hline Wage \& salary & 39,381 & 37,911 & 39,110 & 42,268 & 44,283 & 46,649 & 47,450 & 45,170 \\
\hline Self-employed & 8,250 & 8,837 & 9,050 & 10,786 & 9,917 & 10,324 & 10,300 & 10,310 \\
\hline Payroll tax (nonprofit) & 1,130 & 1,286 & 1,240 & 1,271 & 1,700 & 1,209 & 1,750 & 1,690 \\
\hline Taxes on property & 3,230 & 3,773 & 3,740 & 3,836 & 3,850 & 2,990 & 2,750 & 2,840 \\
\hline Real-estate purchase tax & 1,460 & 1,647 & 2,130 & 2,003 & 2,500 & 1,701 & 1,600 & 1,400 \\
\hline Land betterment tax & 970 & 1,005 & 1,240 & 1,334 & 1,300 & 1,081 & 1,000 & 1,140 \\
\hline Property tax & 800 & 1,121 & 370 & 500 & 50 & 207 & 150 & 300 \\
\hline Taxes on goods and services & 61,359 & 59,478 & 65,710 & 62,939 & 70,770 & 63,484 & 70,280 & 77,936 \\
\hline VAT & 44,209 & 41,463 & 46,620 & 43,849 & 51,170 & 45,452 & 51,480 & 57,036 \\
\hline Domestic expenditure \& civilian imports & 36,869 & 36,414 & 38,870 & 38,515 & 42,600 & 39,373 & 42,500 & 47,300 \\
\hline Nonprofit entities & 5,050 & 3,234 & 5,400 & 3,433 & 6,050 & 3,147 & 6,000 & 6,310 \\
\hline Financial institutions & 1,380 & 925 & 1,190 & 759 & 1,400 & 1,520 & 1,700 & 1,740 \\
\hline Defense imports & 910 & 890 & 1,160 & 1,142 & 1,120 & 1,412 & 1,280 & 1,686 \\
\hline Excises & 7,360 & 7,379 & 8,440 & 7,597 & 8,200 & 7,472 & 7,800 & 9,440 \\
\hline Fuel & 5,650 & 5,661 & 6,570 & 5,666 & 6,100 & 5,701 & 5,950 & 7,310 \\
\hline Tobacco & 910 & 858 & 1,010 & 929 & 950 & 900 & 950 & 1,150 \\
\hline Stamp & 800 & 860 & 860 & 1,003 & 1,150 & 871 & 900 & 980 \\
\hline Purchase tax & 8,610 & 9,291 & 9,310 & 10,072 & 9,800 & 9,204 & 9,600 & 10,020 \\
\hline Domestic & 810 & 805 & 860 & 662 & 800 & 384 & 400 & 450 \\
\hline Imports & 7,800 & 8,486 & 8,450 & 9,409 & 9,000 & 8,819 & 9,200 & 9,570 \\
\hline Customs duties & 1,180 & 1,345 & 1,340 & 1,421 & 1,600 & 1,357 & 1,400 & 1,440 \\
\hline Adjustment to budget frame & 0 & 0 & 0 & 0 & 0 & & 0 & \\
\hline Total nontax revenue & 31,798 & 28,488 & 32,907 & 28,757 & 31,515 & 24,164 & 34,467 & 37,053 \\
\hline Interest & 3,540 & 2,801 & 3,490 & 2,957 & 2,711 & 2,914 & 2,760 & 2,609 \\
\hline Domestic & 2,828 & 2,518 & 2,660 & 2,570 & 2,566 & 2,350 & 2,613 & 2,433 \\
\hline Foreign & 712 & 283 & 830 & 387 & 146 & 564 & 147 & 176 \\
\hline Loans from National Insurance Institute & 6,320 & 5,850 & 5,490 & 7,120 & 6,834 & 4,100 & 9,687 & 12,644 \\
\hline Fees, royalties, pension provisions, \& misc. & 12,571 & 10,559 & 13,223 & 6,697 & 8,748 & 6,654 & 8,917 & 7,623 \\
\hline Income from Israel Land Administration & 1,201 & 1,383 & 1,050 & 1,459 & 1,105 & 277 & 788 & 1,151 \\
\hline Revenue for revenue-dependent expenditure & 8,167 & 7,896 & 9,654 & 10,524 & 12,117 & 10,219 & 12,316 & 13,026 \\
\hline Total revenue & 158,248 & 153,496 & 165,757 & 172,393 & 181,135 & 167,958 & 185,497 & 192,239 \\
\hline Foreign grants & 11,808 & 11,049 & 12,260 & 11,588 & 11,466 & 9,755 & 12,281 & 13,464 \\
\hline Total revenue and grants & 170,056 & 164,545 & 178,017 & 183,981 & 192,601 & 177,713 & 197,778 & 205,703 \\
\hline \multicolumn{9}{|l|}{ Memorandum items: } \\
\hline Domestic revenue & 155,990 & 152,323 & 163,131 & 170,864 & 179,869 & 165,973 & 179,652 & 185,070 \\
\hline Foreign revenue and grants & 14,066 & 12,222 & 14,886 & 13,117 & 12,732 & 11,740 & 18,126 & 20,633 \\
\hline & \multicolumn{8}{|c|}{ (In percent of GDP) } \\
\hline Total tax revenue & 29.6 & 26.9 & 28.6 & 30.6 & 31.8 & 29.8 & 31.3 & 32.1 \\
\hline Taxes on income and profits & 14.2 & 13.0 & 13.4 & 16.1 & 15.6 & 15.7 & 15.8 & 15.0 \\
\hline Companies & 3.1 & 3.0 & 3.0 & 4.8 & 4.1 & 4.0 & 3.8 & 3.6 \\
\hline Individuals & 11.2 & 10.1 & 10.4 & 11.3 & 11.5 & 11.8 & 11.9 & 11.5 \\
\hline Payroll tax (nonprofit) & 0.3 & 0.3 & 0.3 & 0.3 & 0.4 & 0.3 & 0.4 & 0.3 \\
\hline Taxes on property & 0.8 & 0.8 & 0.8 & 0.8 & 0.8 & 0.6 & 0.6 & 0.6 \\
\hline Taxes on goods and services & 14.4 & 12.8 & 14.1 & 13.4 & 15.1 & 13.1 & 14.5 & 16.1 \\
\hline VAT & 10.4 & 8.9 & 10.0 & 9.3 & 10.9 & 9.4 & 10.7 & 11.8 \\
\hline Excises & 1.7 & 1.6 & 1.8 & 1.6 & 1.7 & 1.5 & 1.6 & 2.0 \\
\hline Purchase tax & 2.0 & 2.0 & 2.0 & 2.1 & 2.1 & 1.9 & 2.0 & 2.1 \\
\hline Customs duties & 0.3 & 0.3 & 0.3 & 0.3 & 0.3 & 0.3 & 0.3 & 0.3 \\
\hline Adjustment to budget frame & 0.0 & 0.0 & 0.0 & 0.0 & 0.0 & 0.0 & 0.0 & 0.0 \\
\hline Total nontax revenue & 7.4 & 6.1 & 7.1 & 6.1 & 6.7 & 5.0 & 7.1 & 7.7 \\
\hline Total revenue & 37.1 & 33.1 & 35.7 & 36.7 & 38.5 & 34.8 & 38.4 & 39.8 \\
\hline Foreign grants & 2.8 & 2.4 & 2.6 & 2.5 & 2.4 & 2.0 & 2.5 & 2.8 \\
\hline Total revenue and grants & 39.8 & 35.4 & 38.3 & 39.1 & 41.0 & 36.8 & 40.9 & 42.6 \\
\hline \multicolumn{9}{|l|}{ Memorandum items: } \\
\hline Domestic revenue & 36.5 & 32.8 & 35.1 & 36.3 & 38.3 & 34.3 & 37.2 & 38.3 \\
\hline Foreign revenue and grants & 3.3 & 2.6 & 3.2 & 2.8 & 2.7 & 2.4 & 3.8 & 4.3 \\
\hline
\end{tabular}


Table 25. Israel: State Budget Expenditure (Economic Classification) 1999-2003

\begin{tabular}{|c|c|c|c|c|c|c|c|c|}
\hline & $\begin{array}{r}1999 \\
\text { Budget } \\
\end{array}$ & $\begin{array}{r}1999 \\
\text { Outturn } \\
\end{array}$ & $\begin{array}{r}2000 \\
\text { Budget } \\
\end{array}$ & $\begin{array}{r}2000 \\
\text { Outturn } \\
\end{array}$ & $\begin{array}{r}2001 \\
\text { Budget } \\
\end{array}$ & $\begin{array}{r}2001 \\
\text { Outturn } \\
\end{array}$ & $\begin{array}{c}2002 \\
\text { Budget }\end{array}$ & $\begin{array}{r}2003 \\
\text { Budget } \\
\end{array}$ \\
\hline & \multicolumn{8}{|c|}{ (In millions of new sheqalim) } \\
\hline Current expenditure & 161,761 & 167,870 & 174,810 & 179,448 & 186,057 & 190,442 & 198,524 & 205,737 \\
\hline Wages & 33,943 & 35,265 & 38,458 & 41,234 & 39,405 & 41,062 & 43,007 & 44,375 \\
\hline Of which: defense & 12,860 & 13,287 & 14,746 & 18,113 & 14,894 & 15,628 & 16,540 & 16,358 \\
\hline Goods and services & 33,230 & 34,643 & 36,914 & 38,230 & 40,841 & 43,340 & 43,364 & 45,981 \\
\hline Of which: defense & 21,072 & 22,422 & 22,782 & 24,738 & 26,172 & 29,031 & 27,980 & 29,567 \\
\hline Interest & 23,836 & 24,930 & 26,339 & 27,748 & 28,570 & 27,906 & 28,957 & 33,250 \\
\hline Domestic & 18,131 & 19,125 & 19,883 & 21,559 & 21,481 & 21,824 & 22,519 & 24,623 \\
\hline Foreign & 5,705 & 5,805 & 6,456 & 6,189 & 7,089 & 6,082 & 6,438 & 8,627 \\
\hline Subsidies and transfers & 66,011 & 68,283 & 68,004 & 67,316 & 71,837 & 72,905 & 77,169 & 75,959 \\
\hline Subsidies & 5,544 & 5,910 & 5,741 & 5,016 & 5,253 & 4,829 & 5,379 & 5,567 \\
\hline Nonexport & 1,448 & 1,636 & 1,842 & 1,532 & 1,881 & 1,711 & 2,132 & 2,312 \\
\hline Export & 0 & 0 & 0 & 0 & 0 & 0 & 0 & 0 \\
\hline Investment grants & 4,096 & 4,274 & 3,899 & 3,484 & 3,372 & 3,118 & 3,247 & 3,255 \\
\hline Transfers & 60,468 & 62,373 & 62,263 & 62,299 & 66,583 & 68,077 & 71,790 & 70,392 \\
\hline To local authorities & 9,059 & 9,708 & 8,891 & 9,803 & 9,752 & 10,645 & 10,096 & 10,011 \\
\hline To National Insurance Institute & 16,684 & 16,893 & 18,134 & 18,240 & 20,696 & 21,230 & 22,640 & 21,057 \\
\hline To nonprofit institutions & 32,118 & 32,071 & 33,765 & 32,926 & 34,658 & 34,128 & 37,148 & 37,404 \\
\hline To defense & 2,606 & 3,701 & 1,474 & 1,331 & 1,477 & 2,073 & 1,906 & 1,920 \\
\hline Repayment to National Insurance Institute & 4,741 & 4,749 & 5,095 & 4,921 & 5,404 & 5,229 & 6,028 & 6,172 \\
\hline Capital expenditure & 9,164 & 7,145 & 9,743 & 7,770 & 10,353 & 8,268 & 10,579 & 10,693 \\
\hline Of which: housing & 2,076 & 1,777 & 2,367 & 1,726 & 2,465 & 1,825 & 2,246 & 2,265 \\
\hline Reserve & 7,131 & 0 & 4,374 & 0 & 4,591 & 0 & 3,325 & 4,473 \\
\hline Total expenditure & 178,056 & 175,016 & 188,927 & 187,218 & 201,001 & 198,710 & 212,428 & 220,903 \\
\hline \multicolumn{9}{|l|}{ Memorandum items: } \\
\hline Domestic expenditure & 161,975 & 160,346 & 170,625 & 173,369 & 182,377 & 182,886 & 192,636 & 196,628 \\
\hline \multirow[t]{2}{*}{ Foreign expenditure } & 16,081 & 14,670 & 18,302 & 13,849 & 18,625 & 15,824 & 19,792 & 24,275 \\
\hline & \multicolumn{8}{|c|}{ (In percent of GDP) } \\
\hline Current expenditure & 37.9 & 36.1 & 37.6 & 38.2 & 39.6 & 39.4 & 41.1 & 42.6 \\
\hline Wages & 8.0 & 7.6 & 8.3 & 8.8 & 8.4 & 8.5 & 8.9 & 9.2 \\
\hline Goods and services & 7.8 & 7.5 & 7.9 & 8.1 & 8.7 & 9.0 & 9.0 & 9.5 \\
\hline Interest & 5.6 & 5.4 & 5.7 & 5.9 & 6.1 & 5.8 & 6.0 & 6.9 \\
\hline Domestic & 4.2 & 4.1 & 4.3 & 4.6 & 4.6 & 4.5 & 4.7 & 5.1 \\
\hline Foreign & 1.3 & 1.2 & 1.4 & 1.3 & 1.5 & 1.3 & 1.3 & 1.8 \\
\hline Subsidies and transfers & 15.5 & 14.7 & 14.6 & 14.3 & 15.3 & 15.1 & 16.0 & 15.7 \\
\hline Repayment to National Insurance Institute & 1.1 & 1.0 & 1.1 & 1.0 & 1.1 & 1.1 & 1.2 & 1.3 \\
\hline Capital expenditure & 2.1 & 1.5 & 2.1 & 1.7 & 2.2 & 1.7 & 2.2 & 2.2 \\
\hline Of which : housing & 0.5 & 0.4 & 0.5 & 0.4 & 0.5 & 0.4 & 0.5 & 0.5 \\
\hline Reserve & -- & -- & -- & -- & -- & -- & -- & -- \\
\hline Total expenditure & 41.7 & 37.7 & 40.7 & 39.8 & 42.8 & 41.1 & 44.0 & 45.7 \\
\hline \multicolumn{9}{|l|}{ Memorandum items: } \\
\hline Domestic expenditure & 37.9 & 34.5 & 36.7 & 36.9 & 38.8 & 37.8 & 39.9 & 40.7 \\
\hline Foreign expenditure & 3.8 & 3.2 & 3.9 & 2.9 & 4.0 & 3.3 & 4.1 & 5.0 \\
\hline
\end{tabular}

Source: Data provided by the Ministry of Finance. 
Table 26. Israel: State Budget Expenditure (Functional Classification), 1999-2003

\begin{tabular}{|c|c|c|c|c|c|c|c|c|}
\hline & $\begin{array}{r}1999 \\
\text { Budget }\end{array}$ & $\begin{array}{r}1999 \\
\text { Outturn }\end{array}$ & $\begin{array}{r}2000 \\
\text { Budget }\end{array}$ & $\begin{array}{r}2000 \\
\text { Outturn }\end{array}$ & $\begin{array}{r}2001 \\
\text { Budget }\end{array}$ & $\begin{array}{r}2001 \\
\text { Outturn }\end{array}$ & $\begin{array}{r}2002 \\
\text { Budget }\end{array}$ & $\begin{array}{c}2003 \\
\text { Budget }\end{array}$ \\
\hline & \multicolumn{8}{|c|}{ (In millions of new sheqalim) } \\
\hline General public service & 15,731 & 15,708 & 17,674 & 17,390 & 18,967 & 18,766 & 20,188 & 22,480 \\
\hline General administration & 10,466 & 10,399 & 11,781 & 11,443 & 12,692 & 12,403 & 13,236 & 14,734 \\
\hline Public order & 5,265 & 5,309 & 5,893 & 5,947 & 6,274 & 6,363 & 6,952 & 7,745 \\
\hline Defense & 36,426 & 39,210 & 39,138 & 43,534 & 41,989 & 45,940 & 45,771 & 46,979 \\
\hline Education & 26,258 & 26,838 & 28,013 & 27,983 & 29,367 & 30,205 & 31,173 & 32,186 \\
\hline Of which: Universities & 4,782 & 4,850 & 5,250 & 5,287 & 5,319 & 5,111 & 5,600 & 5,862 \\
\hline Health & 13,839 & 14,308 & 14,685 & 14,130 & 14,617 & 15,284 & 15,128 & 15,849 \\
\hline Labor and welfare & 22,200 & 22,573 & 24,238 & 24,538 & 27,651 & 27,759 & 30,624 & 28,457 \\
\hline Other social services & 14,188 & 12,846 & 15,039 & 12,780 & 14,449 & 13,309 & 14,964 & 14,585 \\
\hline Of which: Housing & 10,186 & 8,831 & 10,799 & 8,385 & 10,125 & 9,086 & 10,503 & 10,504 \\
\hline Immigrant absorption & 1,752 & 1,776 & 1,945 & 1,969 & 2,100 & 1,767 & 1,824 & 1,586 \\
\hline Economic services & 12,115 & 10,603 & 12,826 & 10,965 & 12,737 & 10,989 & 13,677 & 13,507 \\
\hline General & 776 & 923 & 879 & 806 & 847 & 753 & 790 & 677 \\
\hline Agricultural & 1,257 & 1,123 & 1,321 & 1,236 & 1,358 & 1,342 & 1,174 & 1,230 \\
\hline Manufacturing & 4,039 & 4,021 & 3,926 & 3,616 & 3,387 & 3,169 & 3,602 & 3,242 \\
\hline Water and energy & 1,334 & 1,037 & 1,347 & 1,042 & 1,284 & 916 & 1,476 & 1,241 \\
\hline Transport and communications & 3,098 & 3,015 & 3,795 & 3,666 & 4,419 & 4,145 & 5,018 & 5,536 \\
\hline Other & 1,611 & 483 & 1,558 & 599 & 1,441 & 664 & 1,617 & 1,581 \\
\hline Unallocable and other functions & 32,657 & 26,723 & 31,748 & 29,140 & 33,723 & 30,734 & 34,086 & 40,781 \\
\hline Interest & 23,836 & 24,930 & 26,339 & 27,748 & 28,570 & 27,906 & 28,957 & 33,250 \\
\hline Interest and loan subsidy & 29 & 43 & 41 & 48 & 39 & 44 & 24 & 39 \\
\hline Subsidy for basic products & 1,946 & 1,963 & 2,377 & 1,882 & 2,418 & 2,084 & 2,744 & 3,232 \\
\hline Export market development & 0 & 0 & 0 & 0 & 0 & 0 & 0 & 0 \\
\hline General transfers & 4,577 & 4,957 & 4,294 & 4,957 & 4,773 & 4,837 & 4,820 & 4,717 \\
\hline Reserves & 7,417 & 0 & 4,416 & 0 & 4,609 & 0 & 3,334 & 4,480 \\
\hline Repayment of loans (-) & $-5,147$ & $-5,170$ & $-5,720$ & $-5,495$ & $-6,684$ & $-4,137$ & $-5,791$ & $-4,937$ \\
\hline Total expenditure and net lending & 173,413 & 168,808 & 183,361 & $\begin{array}{l}180,460 \\
\text { (Percent }\end{array}$ & $\begin{array}{l}193,499 \\
\text { ( GDP) }\end{array}$ & 192,985 & 205,611 & 214,822 \\
\hline General public service & 3.7 & 3.4 & 3.8 & 3.7 & 4.0 & 3.9 & 4.2 & 4.7 \\
\hline Defense & 8.5 & 8.4 & 8.4 & 9.3 & 8.9 & 9.5 & 9.5 & 9.7 \\
\hline Education & 6.2 & 5.8 & 6.0 & 6.0 & 6.2 & 6.2 & 6.5 & 6.7 \\
\hline Health & 3.2 & 3.1 & 3.2 & 3.0 & 3.1 & 3.2 & 3.1 & 3.3 \\
\hline Labor and welfare & 5.2 & 4.9 & 5.2 & 5.2 & 5.9 & 5.7 & 6.3 & 5.9 \\
\hline Other social services & 3.3 & 2.8 & 3.2 & 2.7 & 3.1 & 2.8 & 3.1 & 3.0 \\
\hline Of which: Housing & 2.4 & 1.9 & 2.3 & 1.8 & 2.2 & 1.9 & 2.2 & 2.2 \\
\hline Immigrant absorption & 0.4 & 0.4 & 0.4 & 0.4 & 0.4 & 0.4 & 0.4 & 0.3 \\
\hline Economic services & 2.8 & 2.3 & 2.8 & 2.3 & 2.7 & 2.3 & 2.8 & 2.8 \\
\hline Unallocable and other functions & 7.7 & 5.8 & 6.8 & 6.2 & 7.2 & 6.4 & 7.1 & 8.4 \\
\hline Interest & 5.6 & 5.4 & 5.7 & 5.9 & 6.1 & 5.8 & 6.0 & 6.9 \\
\hline Interest and loan subsidy & 0.0 & 0.0 & 0.0 & 0.0 & 0.0 & 0.0 & 0.0 & 0.0 \\
\hline Subsidy for basic products & 0.5 & 0.4 & 0.5 & 0.4 & 0.5 & 0.4 & 0.6 & 0.7 \\
\hline Export market development & 0.0 & 0.0 & 0.0 & 0.0 & 0.0 & 0.0 & 0.0 & 0.0 \\
\hline General transfers & 1.1 & 1.1 & 0.9 & 1.1 & 1.0 & 1.0 & 1.0 & 1.0 \\
\hline Reserves & 1.7 & 0.0 & 1.0 & 0.0 & 1.0 & 0.0 & 0.7 & 0.9 \\
\hline Repayment of loans (-) & -1.2 & -1.1 & -1.2 & -1.2 & -1.4 & -0.9 & -1.2 & -1.0 \\
\hline Total expenditure and net lending & 40.6 & 36.3 & 39.5 & 38.4 & 41.2 & 39.9 & 42.5 & 44.4 \\
\hline
\end{tabular}

Source: Data provided by the Ministry of Finance. 
Table 27. Israel: General Government Balance and Financing (National Accounts), 1997-2003

\begin{tabular}{|c|c|c|c|c|c|c|c|}
\hline & 1997 & 1998 & 1999 & 2000 & 2001 & 2002 & 2003 \\
\hline & & & & & & & Est. \\
\hline & \multicolumn{7}{|c|}{ (In millions of new sheqalim) } \\
\hline Total receipts (including foreign receipts) & 174,547 & 193,839 & 205,766 & 228,963 & 235,718 & 249,014 & 239,659 \\
\hline Total expenditure (including foreign expenditure) & 190,692 & 208,271 & 223,606 & 239,026 & 255,019 & 271,249 & 271,475 \\
\hline Overall balance & $-16,145$ & $-14,432$ & $-17,840$ & $-10,063$ & $-19,301$ & $-22,235$ & $-31,816$ \\
\hline Total financing $1 /$ & 16,145 & 14,432 & 17,840 & 10,063 & 19,301 & 22,235 & 31,816 \\
\hline Change in monetary base & 3,671 & 241 & 3,927 & 312 & 4,364 & 1,437 & 3,567 \\
\hline Foreign borrowing (net) & $-1,562$ & $-1,494$ & -281 & -751 & $-2,918$ & $-2,000$ & 6,727 \\
\hline Domestic borrowing (net) & 7,687 & 11,610 & 12,766 & 7,605 & 17,683 & 22,398 & 21,252 \\
\hline Through government (net) & 784 & 3,995 & 7,463 & -409 & 16,762 & 24,400 & 22,488 \\
\hline Other & 6,903 & 7,615 & 5,304 & 8,013 & 921 & $-2,002$ & $-1,236$ \\
\hline Sale of assets & 6,350 & 4,075 & 1,427 & 2,898 & 172 & 400 & 270 \\
\hline \multicolumn{8}{|l|}{ Memorandum items: } \\
\hline Current balance (net saving) & $-10,606$ & $-10,314$ & $-13,565$ & $-7,249$ & $-16,024$ & $-19,421$ & $-31,149$ \\
\hline Current balance + depreciation (gross saving) & $-5,654$ & $-4,725$ & $-7,251$ & -538 & $-8,896$ & $-11,425$ & $-22,433$ \\
\hline Domestic balance & $-20,188$ & $-19,649$ & $-22,668$ & $-16,625$ & $-25,046$ & $-27,459$ & $-35,577$ \\
\hline Foreign balance & 4,043 & 5,217 & 4,828 & 6,562 & 5,744 & 5,224 & 3,761 \\
\hline GDP & \multicolumn{7}{|c|}{ (In percent of GDP) } \\
\hline Total receipts (including foreign receipts) & 49.0 & 49.3 & 47.9 & 48.9 & 49.7 & 50.7 & 48.4 \\
\hline Total expenditure (including foreign expenditure) & 53.6 & 53.0 & 52.1 & 51.1 & 53.8 & 55.2 & 54.8 \\
\hline Overall balance & -4.5 & -3.7 & -4.2 & -2.1 & -4.1 & -4.5 & -6.4 \\
\hline Total financing & 4.5 & 3.7 & 4.2 & 2.1 & 4.1 & 4.5 & 6.4 \\
\hline Change in monetary base & 1.0 & 0.1 & 0.9 & 0.1 & 0.9 & 0.3 & 0.7 \\
\hline Foreign borrowing (net) & -0.4 & -0.4 & -0.1 & -0.2 & -0.6 & -0.4 & 1.4 \\
\hline Domestic borrowing (net) & 2.2 & 3.0 & 3.0 & 1.6 & 3.7 & 4.6 & 4.3 \\
\hline Through government (net) & 0.2 & 1.0 & 1.7 & -0.1 & 3.5 & 5.0 & 4.5 \\
\hline Through Bank of Israel (net) & 1.9 & 1.9 & 1.2 & 1.7 & 0.2 & -0.4 & -0.2 \\
\hline Sale of assets & 1.8 & 1.0 & 0.3 & 0.6 & 0.0 & 0.1 & 0.1 \\
\hline \multicolumn{8}{|l|}{ Memorandum items: } \\
\hline Current balance (net saving) & -3.0 & -2.6 & -3.2 & -1.5 & -3.4 & -4.0 & -6.3 \\
\hline Current balance + depreciation (gross saving) & -1.6 & -1.2 & -1.7 & -0.1 & -1.9 & -2.3 & -4.5 \\
\hline Domestic balance & -5.7 & -5.0 & -5.3 & -3.6 & -5.3 & -5.6 & -7.2 \\
\hline Foreign balance & 1.1 & 1.3 & 1.1 & 1.4 & 1.2 & 1.1 & 0.8 \\
\hline
\end{tabular}

Sources: Bank of Israel; and revised BCS data from January 2004.

$1 /$ The finance figures relate only to the central government. 
Table 28. Israel: General Government Receipts (National Accounts), 1997-2003

\begin{tabular}{|c|c|c|c|c|c|c|c|}
\hline & 1997 & 1998 & 1999 & 2000 & 2001 & 2002 & $\frac{2003}{\text { Est }}$ \\
\hline & \multicolumn{7}{|c|}{ (In millions of new sheqalim) } \\
\hline Total domestic receipts & 157,505 & 173,511 & 183,887 & 206,637 & 213,275 & 224,051 & 217,707 \\
\hline Current receipts & 144,840 & 159,049 & 169,604 & 191,160 & 197,410 & 206,327 & 199,792 \\
\hline Taxes and fees & 137,454 & 148,464 & 162,663 & 183,939 & 187,329 & 190,411 & 189,976 \\
\hline Indirect taxes & 65,710 & 70,291 & 77,672 & 81,483 & 82,225 & 88,416 & 90,545 \\
\hline Domestic production & 49,340 & 53,466 & 58,579 & 59,889 & 62,157 & 67,908 & 70,013 \\
\hline Civilian imports & 16,370 & 16,825 & 19,093 & 21,594 & 20,068 & 20,508 & 20,532 \\
\hline Direct taxes and fees & 51,230 & 55,560 & 60,335 & 74,828 & 75,376 & 70,618 & 66,867 \\
\hline National insurance income 1/ & 20,514 & 22,613 & 24,656 & 27,628 & 29,728 & 31,377 & 32,564 \\
\hline Of which: health tax & 7,140 & 7,874 & 8,725 & 9,762 & 10,577 & 10,788 & 10,949 \\
\hline Transfer payments & 5,614 & 6,047 & 6,466 & 6,945 & 7,376 & 7,862 & 7,766 \\
\hline Of which: imputed pensions & 5,481 & 5,612 & 5,695 & 6,034 & 6,370 & 6,416 & 6,750 \\
\hline Property income/tax & 1,772 & 4,538 & 475 & 276 & 2,705 & 8,054 & 2,050 \\
\hline Capital receipts & 12,665 & 14,462 & 14,283 & 15,477 & 15,865 & 17,724 & 17,915 \\
\hline Transfer payments & 7,713 & 8,873 & 7,969 & 8,766 & 8,736 & 9,728 & 9,199 \\
\hline Depreciation & 4,952 & 5,589 & 6,314 & 6,711 & 7,129 & 7,996 & 8,716 \\
\hline Total foreign receipts & 17,042 & 20,328 & 21,879 & 22,326 & 22,443 & 24,963 & 21,952 \\
\hline Current receipts & 16,434 & 19,630 & 21,205 & 21,673 & 21,760 & 24,125 & 21,286 \\
\hline Interest & 2,707 & 4,012 & 4,205 & 4,562 & 4,352 & 3,954 & 2,695 \\
\hline Transfer payments & 13,727 & 15,618 & 17,000 & 17,111 & 17,408 & 20,171 & 18,591 \\
\hline Intergovernmental & 11,283 & 12,659 & 13,935 & 13,268 & 14,331 & 16,424 & 14,267 \\
\hline National and nonprofit institutions & 2,444 & 2,959 & 3,065 & 3,843 & 3,077 & 3,747 & 4,324 \\
\hline Capital receipts & 608 & 698 & 674 & 653 & 683 & 838 & 666 \\
\hline Total receipts & 174,547 & 193,839 & 205,766 & 228,963 & 235,718 & 249,014 & 239,659 \\
\hline \multirow[t]{2}{*}{ GDP } & 356,055 & 393,104 & 429,542 & 468,144 & 474,043 & 491,260 & 495,360 \\
\hline & \multicolumn{7}{|c|}{ (In percent of GDP) } \\
\hline Total domestic receipts & 44.2 & 44.1 & 42.8 & 44.1 & 45.0 & 45.6 & 43.9 \\
\hline Current receipts & 40.7 & 40.5 & 39.5 & 40.8 & 41.6 & 42.0 & 40.3 \\
\hline Taxes and fees & 38.6 & 37.8 & 37.9 & 39.3 & 39.5 & 38.8 & 38.4 \\
\hline Indirect taxes & 18.5 & 17.9 & 18.1 & 17.4 & 17.3 & 18.0 & 18.3 \\
\hline Domestic production & 13.9 & 13.6 & 13.6 & 12.8 & 13.1 & 13.8 & 14.1 \\
\hline Civilian imports & 4.6 & 4.3 & 4.4 & 4.6 & 4.2 & 4.2 & 4.1 \\
\hline Direct taxes and fees & 14.4 & 14.1 & 14.0 & 16.0 & 15.9 & 14.4 & 13.5 \\
\hline National insurance income 1/ & 5.8 & 5.8 & 5.7 & 5.9 & 6.3 & 6.4 & 6.6 \\
\hline Transfer payments & 1.6 & 1.5 & 1.5 & 1.5 & 1.6 & 1.6 & 1.6 \\
\hline Of which: Imputed pensions & 1.5 & 1.4 & 1.3 & 1.3 & 1.3 & 1.3 & 1.4 \\
\hline Property income/tax & 0.5 & 1.2 & 0.1 & 0.1 & 0.6 & 1.6 & 0.4 \\
\hline Capital receipts & 3.6 & 3.7 & 3.3 & 3.3 & 3.3 & 3.6 & 3.6 \\
\hline Transfer payments & 2.2 & 2.3 & 1.9 & 1.9 & 1.8 & 2.0 & 1.9 \\
\hline Depreciation & 1.4 & 1.4 & 1.5 & 1.4 & 1.5 & 1.6 & 1.8 \\
\hline Total foreign receipts & 4.8 & 5.2 & 5.1 & 4.8 & 4.7 & 5.1 & 4.4 \\
\hline Current receipts & 4.6 & 5.0 & 4.9 & 4.6 & 4.6 & 4.9 & 4.3 \\
\hline Interest & 0.8 & 1.0 & 1.0 & 1.0 & 0.9 & 0.8 & 0.5 \\
\hline Transfer payments & 3.9 & 4.0 & 4.0 & 3.7 & 3.7 & 4.1 & 3.8 \\
\hline Intergovernmental & 3.2 & 3.2 & 3.2 & 2.8 & 3.0 & 3.3 & 2.9 \\
\hline National and nonprofit institutions & 0.7 & 0.8 & 0.7 & 0.8 & 0.6 & 0.8 & 0.9 \\
\hline Capital receipts & 0.2 & 0.2 & 0.2 & 0.1 & 0.1 & 0.2 & 0.1 \\
\hline Total receipts & 49.0 & 49.3 & 47.9 & 48.9 & 49.7 & 50.7 & 48.4 \\
\hline
\end{tabular}

Sources: Bank of Israel; and revised BCS data from January 2004.

1/ Figures from 1995 onward include revenue from the health tax. 
Table 29. Israel: General Government Expenditure (National Accounts), 1997-2003

\begin{tabular}{|c|c|c|c|c|c|c|c|}
\hline & \multirow[t]{2}{*}{1997} & \multirow[t]{2}{*}{1998} & \multirow[t]{2}{*}{1999} & \multirow[t]{2}{*}{2000} & \multirow[t]{2}{*}{2001} & \multirow[t]{2}{*}{2002} & \multirow{2}{*}{$\frac{2003}{\text { Est. }}$} \\
\hline & & & & & & & \\
\hline & \multicolumn{7}{|c|}{ (In millions of new sheqalim) } \\
\hline Total domestic expenditure & 177,692 & 193,160 & 206,555 & 223,262 & 238,320 & 251,510 & 253,284 \\
\hline Current expenditure & 158,881 & 173,882 & 187,324 & 204,318 & 218,495 & 230,134 & 234,036 \\
\hline Civilian 1/ & 70,192 & 76,014 & 82,742 & 89,001 & 96,207 & 103,085 & 103,119 \\
\hline Defense & 25,024 & 26,762 & 28,555 & 31,589 & 31,918 & 36,127 & 36,195 \\
\hline Transfer payments & 44,338 & 49,966 & 54,525 & 59,699 & 66,169 & 68,755 & 68,530 \\
\hline Subsidies & 3,272 & 3,322 & 3,478 & 3,677 & 3,772 & 3,354 & 4,142 \\
\hline Credit & 190 & 138 & 66 & 47 & 44 & 25 & 23 \\
\hline Other & 3,082 & 3,184 & 3,412 & 3,630 & 3,728 & 3,329 & 4,119 \\
\hline Interest & 16,055 & 17,817 & 18,023 & 20,351 & 20,429 & 18,814 & 22,050 \\
\hline Capital expenditure & 18,812 & 19,278 & 19,231 & 18,944 & 19,825 & 21,376 & 19,248 \\
\hline Investment & 11,750 & 12,841 & 11,748 & 12,104 & 12,807 & 13,257 & 13,793 \\
\hline Capital grants & 7,010 & 6,388 & 7,445 & 6,826 & 6,969 & 8,093 & 5,429 \\
\hline Repayment of compulsory loans & 52 & 49 & 38 & 14 & 49 & 26 & 26 \\
\hline Total foreign (current) expenditure & 12,999 & 15,111 & 17,051 & 15,764 & 16,699 & 19,739 & 18,191 \\
\hline Defense imports & 5,727 & 6,824 & 8,210 & 7,057 & 8,200 & 10,671 & 8,835 \\
\hline Interest & 6,374 & 7,199 & 7,635 & 7,565 & 7,330 & 7,763 & 7,952 \\
\hline Other & 898 & 1,088 & 1,206 & 1,143 & 1,170 & 1,304 & 1,404 \\
\hline Total expenditure & 190,692 & 208,271 & 223,606 & 239,026 & 255,019 & 271,249 & 271,475 \\
\hline GDP & \multicolumn{7}{|c|}{ (In percent of GDP) } \\
\hline Total domestic expenditure & 49.9 & 49.1 & 48.1 & 47.7 & 50.3 & 51.2 & 51.1 \\
\hline Current expenditure & 44.6 & 44.2 & 43.6 & 43.6 & 46.1 & 46.8 & 47.2 \\
\hline Civilian 1/ & 19.7 & 19.3 & 19.3 & 19.0 & 20.3 & 21.0 & 20.8 \\
\hline Defense & 7.0 & 6.8 & 6.6 & 6.7 & 6.7 & 7.4 & 7.3 \\
\hline Transfer payments & 12.5 & 12.7 & 12.7 & 12.8 & 14.0 & 14.0 & 13.8 \\
\hline Subsidies & 0.9 & 0.8 & 0.8 & 0.8 & 0.8 & 0.7 & 0.8 \\
\hline Credit & 0.1 & 0.0 & 0.0 & 0.0 & 0.0 & 0.0 & 0.0 \\
\hline Other & 0.9 & 0.8 & 0.8 & 0.8 & 0.8 & 0.7 & 0.8 \\
\hline Interest & 4.5 & 4.5 & 4.2 & 4.3 & 4.3 & 3.8 & 4.5 \\
\hline Capital expenditure & 5.3 & 4.9 & 4.5 & 4.0 & 4.2 & 4.4 & 3.9 \\
\hline Investment & 3.3 & 3.3 & 2.7 & 2.6 & 2.7 & 2.7 & 2.8 \\
\hline Capital grants & 2.0 & 1.6 & 1.7 & 1.5 & 1.5 & 1.6 & 1.1 \\
\hline Repayment of compulsory loans & 0.0 & 0.0 & 0.0 & 0.0 & 0.0 & 0.0 & 0.0 \\
\hline Total foreign (current) expenditure & 3.7 & 3.8 & 4.0 & 3.4 & 3.5 & 4.0 & 3.7 \\
\hline Defense imports & 1.6 & 1.7 & 1.9 & 1.5 & 1.7 & 2.2 & 1.8 \\
\hline Interest & 1.8 & 1.8 & 1.8 & 1.6 & 1.5 & 1.6 & 1.6 \\
\hline Other & 0.3 & 0.3 & 0.3 & 0.2 & 0.2 & 0.3 & 0.3 \\
\hline Total expenditure & 53.6 & 53.0 & 52.1 & 51.1 & 53.8 & 55.2 & 54.8 \\
\hline
\end{tabular}

Sources: Bank of Israel; and revised BCS data from January 2004.

1/ Figures from 1995 onward include expenditure arising from the Health Law. 
Table 30. Israel: Structure of General Government Finances (National Accounts), 1997-2003

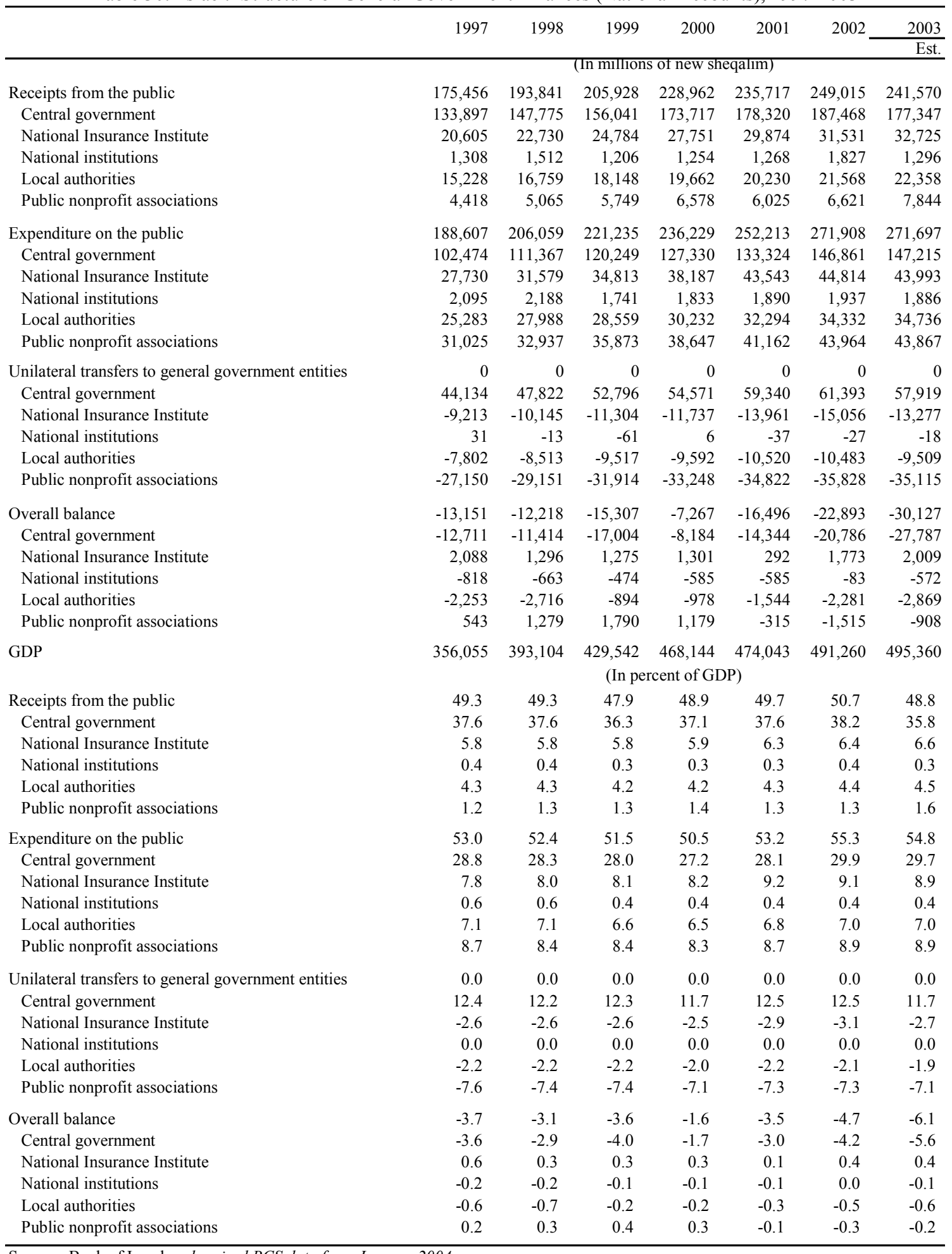

Source: Bank of Israel and revised BCS data from January 2004. 
Table 31. Israel: Currency Basket of the Israeli Sheqel, 1994-2003

\begin{tabular}{|c|c|c|c|c|c|}
\hline & \multicolumn{5}{|c|}{$\begin{array}{l}\text { Percentage distribution of currencies on the } \\
\text { basis of market rates on: }\end{array}$} \\
\hline & $\begin{array}{c}1994 \\
\text { Dec. } 31\end{array}$ & $\begin{array}{c}1995 \\
\text { Dec. } 31\end{array}$ & $\begin{array}{c}1996 \\
\text { Dec. } 31\end{array}$ & $\begin{array}{c}1997 \\
\text { Dec. } 31\end{array}$ & $\begin{array}{c}1998 \\
\text { Dec. } 31\end{array}$ \\
\hline U.S. dollar & 0.5381 & 0.5583 & 0.6028 & 0.6296 & 0.6151 \\
\hline Pound sterling & 0.0939 & 0.0819 & 0.0895 & 0.0909 & 0.0894 \\
\hline Deutschmark & 0.2419 & 0.2424 & 0.2070 & 0.1870 & 0.1954 \\
\hline Japanese yen & 0.0692 & 0.0597 & 0.0505 & 0.0469 & 0.0525 \\
\hline \multirow[t]{3}{*}{ French franc } & 0.0569 & 0.0577 & 0.0502 & 0.0457 & 0.0476 \\
\hline & \multicolumn{5}{|c|}{$\begin{array}{l}\text { Percentage distribution of currencies on the } \\
\text { basis of market rates on: }\end{array}$} \\
\hline & $\begin{array}{l}1999 \\
\text { Dec. } 31\end{array}$ & $\begin{array}{c}2000 \\
\text { Dec. } 31\end{array}$ & $\begin{array}{c}2001 \\
\text { Dec. } 31\end{array}$ & $\begin{array}{c}2002 \\
\text { Dec. } 31\end{array}$ & $\begin{array}{c}2003 \\
\text { Nov. } 21\end{array}$ \\
\hline U.S. dollar & 0.6343 & 0.6487 & 0.6624 & 0.6287 & 0.6035 \\
\hline Pound sterling & 0.0896 & 0.0655 & 0.0649 & 0.0685 & 0.0695 \\
\hline Euro & 0.2159 & 0.2248 & 0.2182 & 0.2455 & 0.2671 \\
\hline Japanese yen & 0.0602 & 0.0610 & 0.0555 & 0.0573 & 0.0599 \\
\hline
\end{tabular}

Sources: IMF, International Financial Statistics; and data provided by the Bank of Israel. 
Table 32. Israel: Exchange Rate Developments, 1997-2003

(Index number $1986=100$; period averages)

\begin{tabular}{|c|c|c|c|c|c|c|}
\hline & $\begin{array}{l}\text { U.S.dollar/ } \\
\text { sheqel }\end{array}$ & $\begin{array}{l}\text { Pound } \\
\text { sterling/ } \\
\text { sheqel }\end{array}$ & $\begin{array}{c}\text { Deutsche } \\
\text { mark/ } \\
\text { sheqel }\end{array}$ & $\begin{array}{c}\text { Japanese } \\
\text { yen/ } \\
\text { sheqel }\end{array}$ & $\begin{array}{l}\text { French } \\
\text { franc/ } \\
\text { sheqel }\end{array}$ & $\begin{array}{c}\text { Currency } \\
\text { basket }\end{array}$ \\
\hline 1997 & 231.9 & 259.2 & 288.6 & 320.9 & 274.5 & 249.9 \\
\hline 1998 & 255.5 & 288.7 & 314.4 & 328.7 & 300.2 & 274.0 \\
\hline 1999 & 278.4 & 307.3 & 327.3 & 411.6 & 312.4 & 296.8 \\
\hline 2000 & 274.1 & 283.1 & 279.2 & 424.8 & 266.6 & 282.6 \\
\hline 2001 & 282.5 & 277.2 & 278.8 & 387.9 & 266.1 & 286.2 \\
\hline 2002 & 318.4 & 326.7 & 332.8 & 426.0 & 317.6 & 327.3 \\
\hline 2003 & 306.5 & 339.2 & 378.8 & 438.7 & 361.5 & 331.1 \\
\hline \multicolumn{7}{|l|}{1997} \\
\hline I & 223.3 & 248.7 & 291.5 & 308.5 & 276.5 & 243.3 \\
\hline II & 229.5 & 256.0 & 288.8 & 320.9 & 274.2 & 248.0 \\
\hline III & 237.1 & 263.1 & 283.3 & 335.8 & 269.3 & 253.2 \\
\hline IV & 237.3 & 269.0 & 291.8 & 316.3 & 278.9 & 254.9 \\
\hline \multicolumn{7}{|l|}{1998} \\
\hline I & 241.0 & 270.5 & 285.9 & 314.3 & 273.1 & 256.2 \\
\hline II & 247.1 & 278.5 & 297.3 & 303.5 & 283.9 & 262.9 \\
\hline III & 250.7 & 282.2 & 306.2 & 298.9 & 292.3 & 267.3 \\
\hline IV & 282.2 & 322.4 & 366.1 & 395.7 & 349.5 & 308.0 \\
\hline \multicolumn{7}{|l|}{1999} \\
\hline I & 272.8 & 304.0 & 338.3 & 391.5 & 322.9 & 294.3 \\
\hline II & 274.9 & 301.2 & 320.0 & 380.1 & 305.5 & 291.2 \\
\hline III & 281.0 & 307.0 & 325.1 & 415.2 & 310.4 & 298.2 \\
\hline IV & 284.1 & 316.1 & 325.5 & 454.4 & 310.8 & 302.7 \\
\hline \multicolumn{7}{|l|}{2000} \\
\hline I & 272.5 & 298.7 & 297.0 & 425.4 & 283.5 & 286.4 \\
\hline II & 275.8 & 287.4 & 283.9 & 431.5 & 271.0 & 285.4 \\
\hline III & 272.8 & 274.9 & 272.5 & 423.3 & 260.2 & 279.8 \\
\hline IV & 275.5 & 271.6 & 263.8 & 419.4 & 251.8 & 279.2 \\
\hline \multicolumn{7}{|l|}{2001} \\
\hline I & 278.0 & 276.6 & 282.9 & 392.7 & 270.0 & 284.3 \\
\hline II & 279.5 & 270.7 & 269.2 & 380.7 & 257.0 & 281.4 \\
\hline III & 285.2 & 279.0 & 279.2 & 390.4 & 266.5 & 288.4 \\
\hline IV & 287.4 & 282.6 & 283.7 & 388.1 & 270.8 & 291.0 \\
\hline \multicolumn{7}{|l|}{2002} \\
\hline I & 310.2 & 301.7 & 300.1 & 391.3 & 286.4 & 311.3 \\
\hline II & 328.1 & 327.2 & 333.0 & 432.4 & 317.8 & 334.1 \\
\hline III & 317.4 & 335.1 & 344.3 & 444.5 & 328.6 & 330.5 \\
\hline IV & 317.8 & 340.6 & 350.5 & 433.1 & 334.6 & 332.1 \\
\hline \multicolumn{7}{|l|}{2003} \\
\hline I & 324.6 & 355.0 & 384.4 & 455.8 & 366.9 & 346.1 \\
\hline II & 301.3 & 333.2 & 378.1 & 424.3 & 360.9 & 326.4 \\
\hline III & 297.6 & 326.5 & 369.0 & 422.7 & 352.2 & 321.4 \\
\hline
\end{tabular}

Sources: IMF, International Financial Statistics; and data provided by the Bank of Israel. 
Table 33. Israel: Balance of Payments, 1998-2003

\begin{tabular}{|c|c|c|c|c|c|c|c|}
\hline & \multirow[t]{2}{*}{1998} & \multirow[t]{2}{*}{1999} & \multirow[t]{2}{*}{2000} & \multirow[t]{2}{*}{2001} & \multirow[t]{2}{*}{2002} & \multicolumn{2}{|c|}{2003} \\
\hline & & & & & & QI & \multirow[t]{2}{*}{ QII } \\
\hline & \multicolumn{6}{|c|}{ (In millions of U.S. dollars) } & \\
\hline Current account balance & $-1,149$ & $-1,512$ & -670 & $-1,776$ & $-1,380$ & 452 & 101 \\
\hline Trade balance & $-3,051$ & $-4,214$ & $-2,883$ & $-3,004$ & $-3,726$ & -486 & -543 \\
\hline Exports f.o.b & 23,190 & 25,827 & 31,153 & 27,974 & 27,486 & 7,417 & 7,027 \\
\hline Imports f.o.b & 26,241 & 30,041 & 34,036 & 30,978 & 31,212 & 7,903 & 7,570 \\
\hline Civillian imports & 24,369 & 27,971 & 32,100 & 28,832 & 28,801 & 7,257 & 7,133 \\
\hline Military imports (defence import: & 1,872 & 2,070 & 1,936 & 2,146 & 2,411 & 645 & 437 \\
\hline Civillan trade balance & $-1,178$ & $-2,144$ & -947 & -858 & $-1,315$ & 159 & -106 \\
\hline Services balance & -146 & 941 & 2,656 & -553 & -605 & 469 & -64 \\
\hline Exports & 9,490 & 11,649 & 15,162 & 11,950 & 10,865 & 2,822 & 2,802 \\
\hline Imports & 9,636 & 10,708 & 12,505 & 12,503 & 11,470 & 2,353 & 2,866 \\
\hline Income (factor income) balance & $-4,028$ & $-4,552$ & $-6,927$ & $-4,627$ & $-3,599$ & -934 & -888 \\
\hline Exports & 2,508 & 2,799 & 3,759 & 2,881 & 2,852 & 570 & 658 \\
\hline Imports & 6,537 & 7,351 & 10,686 & 7,509 & 6,451 & 1,504 & 1,546 \\
\hline Current transfers & 6,076 & 6,313 & 6,483 & 6,409 & 6,549 & 1,404 & 1,595 \\
\hline Capital transfers & 577 & 569 & 455 & 681 & 151 & 86 & 106 \\
\hline Net financial transactions (excluding re & $\ldots$ & 4,600 & 4,054 & 195 & $-1,739$ & -802 & -393 \\
\hline Total net direct investment & $\ldots$ & 2,136 & 1,548 & 2,715 & 417 & 526 & 1,229 \\
\hline Total net portfolio investment & $\ldots$ & 1,918 & 2,228 & $-1,487$ & $-1,909$ & 184 & -474 \\
\hline Total net other investment & $\ldots$ & 696 & -387 & $-1,543$ & -438 & $-1,724$ & $-1,354$ \\
\hline Financial derivatives & $\cdots$ & -149 & 664 & 510 & 191 & 213 & 206 \\
\hline Change in bank of israel total assets anc & $-1,880$ & -929 & -761 & 150 & 746 & 412 & -321 \\
\hline Statistical discrepancies & 588 & $-2,728$ & $-3,077$ & 750 & 2,223 & -149 & 507 \\
\hline & & & (Growth & tes in per & nt) $1 /$ & & \\
\hline \multicolumn{8}{|l|}{ Memorandum items: } \\
\hline Goods exports & 1.4 & 11.4 & 20.6 & -10.2 & -1.7 & 3.1 & -5.3 \\
\hline Goods imports & -5.9 & 14.5 & 13.3 & -9.0 & 0.8 & -5.4 & -4.2 \\
\hline Services exports & 8.7 & 22.7 & 30.2 & -21.2 & -9.1 & -2.9 & -0.7 \\
\hline Services imports & 3.6 & 11.1 & 16.8 & 0.0 & -8.3 & -11.7 & 21.8 \\
\hline
\end{tabular}

Sources: Bank of Israel; and Central Bureau of Statistics.

1/ The growth rate in percent is compared to same period the previous year. 
Table 34. Israel: Balance of Payments--Services, 1997-2003

\begin{tabular}{|c|c|c|c|c|c|c|c|c|}
\hline & \multirow[t]{2}{*}{1997} & \multirow[t]{2}{*}{1998} & \multirow[t]{2}{*}{1999} & \multirow[t]{2}{*}{2000} & \multirow[t]{2}{*}{2001} & \multirow[t]{2}{*}{2002} & \multicolumn{2}{|c|}{2003} \\
\hline & & & & & & & QI & QII \\
\hline & \multicolumn{8}{|c|}{ (In millions of U.S dollars) } \\
\hline Freight and transportation, net & $-1,911$ & $-1,932$ & $-2,028$ & $-2,405$ & $-2,392$ & $-2,078$ & -409 & -483 \\
\hline Receipts & 2,001 & 2,097 & 2,263 & 2,482 & 2,122 & 2,122 & 579 & 614 \\
\hline Payments & $-3,913$ & $-4,028$ & $-4,291$ & $-4,887$ & $-4,514$ & $-4,199$ & -989 & $-1,097$ \\
\hline Travel, net & 1,011 & 684 & 1,739 & 1,348 & -381 & -439 & 82 & -227 \\
\hline Receipts & 3,295 & 3,157 & 4,304 & 4,152 & 2,564 & 2,103 & 473 & 472 \\
\hline Payments & $-2,283$ & $-2,473$ & $-2,566$ & $-2,804$ & $-2,945$ & $-2,543$ & -391 & -698 \\
\hline Insurance, net & -277 & -259 & -323 & -313 & -300 & -364 & -111 & -83 \\
\hline Receipts & 15 & 17 & 18 & 17 & 13 & 15 & 4 & 4 \\
\hline Payments & -292 & -276 & -340 & -330 & -313 & -379 & -116 & -87 \\
\hline Other, net & 712 & 1,483 & 1,666 & 4,142 & 2,680 & 2,450 & 954 & 783 \\
\hline Receipts & 3,348 & 4,143 & 4,965 & 8,428 & 7,209 & 6,598 & 1,758 & 1,706 \\
\hline Payments & $-2,636$ & $-2,660$ & $-3,299$ & $-4,287$ & $-4,529$ & $-4,148$ & -804 & -923 \\
\hline Government, net & -100 & -123 & -113 & -116 & -161 & -174 & -47 & -54 \\
\hline Receipts & 75 & 76 & 99 & 83 & 41 & 27 & 8 & 7 \\
\hline Payments & -175 & -199 & -213 & -198 & -202 & -201 & -54 & -61 \\
\hline Total Services, net & -565 & -146 & 941 & 2,656 & -553 & -605 & 469 & -64 \\
\hline Receipts & 8,734 & 9,490 & 11,649 & 15,162 & 11,950 & 10,865 & 2,822 & 2,802 \\
\hline Payments & $-9,299$ & $-9,636$ & $-10,708$ & $-12,505$ & $-12,503$ & $-11,470$ & $-2,353$ & $-2,866$ \\
\hline Compensation of employees, net & $\ldots$ & $\ldots$ & $-2,938$ & $-3,172$ & $-2,887$ & $-2,505$ & -523 & -475 \\
\hline Receipts & $\ldots$ & $\ldots$ & 154 & 165 & 165 & 150 & 33 & 32 \\
\hline Payments & $\ldots$ & $\ldots$ & 3,092 & 3,337 & 3,052 & 2,655 & 556 & 507 \\
\hline Total investment income, net & $\ldots$ & $\ldots$ & $-1,615$ & $-3,754$ & $-1,740$ & $-1,093$ & -410 & -412 \\
\hline Receipts & $\ldots$ & $\ldots$ & 2,644 & 3,594 & 2,716 & 2,703 & 538 & 626 \\
\hline Payments & $\ldots$ & $\ldots$ & 4,259 & 7,348 & 4,456 & 3,796 & 948 & 1,038 \\
\hline
\end{tabular}

Source: Central Bureau of Statistics. 
Table 35. Israel: Export Volume and Price Indices, 1997-2003 (percentage change from previous period)

\begin{tabular}{|c|c|c|c|c|c|c|c|c|c|}
\hline & \multirow[t]{2}{*}{1997} & \multirow[t]{2}{*}{1998} & \multirow[t]{2}{*}{1999} & \multirow[t]{2}{*}{2000} & \multirow[t]{2}{*}{2001} & \multirow[t]{2}{*}{2002} & \multicolumn{3}{|c|}{2003} \\
\hline & & & & & & & QI & QII & QIII \\
\hline \multicolumn{10}{|l|}{ Volume indicators $1 /$} \\
\hline Agricultural products & 8.5 & 7.1 & 1.7 & -2.6 & -9.4 & -8.6 & 46.1 & -36.5 & -42.9 \\
\hline Citrus fruit & 3.4 & -13.7 & -15.5 & -21.5 & -14.9 & -30.4 & 65.8 & -58.8 & -70.2 \\
\hline Other fruits and vegetables & 0.1 & 19.1 & 5.3 & 10.0 & 7.1 & -4.5 & 76.2 & -49.8 & -59.2 \\
\hline Other & 16.4 & 11.1 & 6.1 & -3.9 & -18.6 & -6.5 & 16.0 & -12.7 & -26.9 \\
\hline Industrial products (excl. diamonds) & 13.9 & 11.6 & 7.1 & 31.6 & -4.8 & -5.6 & -2.9 & -2.1 & 1.8 \\
\hline Ores and minerals & 9.6 & 0.7 & -7.8 & 8.6 & -8.1 & 5.1 & 10.1 & 47.1 & -6.6 \\
\hline Food (incl. Beverages and tobacco) & -12.9 & -5.7 & -9.9 & 3.3 & 1.2 & -0.5 & 11.5 & -3.3 & 2.1 \\
\hline Textiles (incl. Clothing and leather) & -1.6 & 3.7 & 9.4 & 2.1 & -8.5 & -5.1 & -3.1 & -9.8 & 5.8 \\
\hline Other light industry products & -1.0 & 2.2 & 8.3 & 8.7 & -2.7 & 7.9 & -10.1 & 1.2 & 7.5 \\
\hline Chemicals & 17.4 & 12.0 & 5.2 & 13.7 & 13.4 & 10.2 & -9.9 & 11.4 & -7.1 \\
\hline Metals, machinery and electronics & 20.3 & 16.6 & 8.5 & 47.6 & -8.5 & -13.7 & -0.2 & -9.2 & 5.6 \\
\hline Diamonds, net & -0.9 & -13.0 & 20.4 & 9.8 & -14.2 & 21.1 & 25.1 & -27.2 & 23.9 \\
\hline Total, net & 9.5 & 4.7 & 9.2 & 23.8 & -7.0 & 0.0 & 6.3 & -12.9 & 7.5 \\
\hline Total, excluding diamonds & 14.5 & 12.2 & 5.6 & 29.1 & -4.8 & -6.0 & -0.1 & -6.8 & 2.0 \\
\hline \multicolumn{10}{|l|}{ Price indicators $2 /$} \\
\hline Agricultural products & -8.1 & -5.8 & -4.9 & -7.9 & -0.9 & 7.7 & 10.0 & -9.7 & -6.1 \\
\hline Citrus fruit & -11.0 & 4.5 & 5.9 & -12.2 & 4.3 & 2.0 & -9.0 & 0.9 & 1.2 \\
\hline Other fruits and vegetables & -5.0 & -1.8 & -2.4 & -9.8 & -2.9 & 1.9 & -1.7 & -8.1 & -3.0 \\
\hline Other & -9.5 & -12.1 & -9.7 & -5.3 & -0.5 & 14.1 & 30.0 & -16.2 & -9.3 \\
\hline Industrial products (excl. diamonds) & -3.0 & -3.3 & -1.5 & -2.8 & -2.1 & -1.0 & 1.9 & -0.3 & -0.3 \\
\hline Ores and minerals & 5.0 & 3.9 & 2.5 & -0.6 & 1.4 & 1.0 & 5.5 & 1.9 & -3.1 \\
\hline Food (incl. Beverages and tobacco) & -0.5 & -4.3 & 2.5 & -2.2 & -4.8 & 0.9 & -2.1 & 2.9 & 1.3 \\
\hline Textiles (incl. Clothing and leather) & 1.5 & 2.8 & -2.4 & -1.4 & -0.6 & 1.6 & 2.9 & -1.2 & 1.6 \\
\hline Other light industry products & 3.2 & -3.6 & 0.1 & -4.1 & 3.3 & -1.6 & 0.8 & 1.6 & -1.5 \\
\hline Chemicals & -3.2 & -7.6 & -0.3 & 2.2 & -6.7 & -1.2 & 7.5 & -4.9 & 0.9 \\
\hline Metals, machinery and electronics & -4.6 & -3.1 & -2.3 & -4.6 & -1.8 & -0.8 & 0.3 & 0.4 & -0.4 \\
\hline Diamonds, net & 4.3 & -2.0 & 8.5 & 9.2 & -3.1 & 0.9 & -4.6 & 19.0 & -6.9 \\
\hline Total, net & -1.2 & -2.9 & 1.0 & 0.5 & -2.4 & -0.3 & -0.3 & 6.1 & -3.0 \\
\hline Total, excluding diamonds & -4.1 & -4.5 & -0.6 & -2.4 & -2.2 & -0.7 & 1.5 & 1.6 & -1.8 \\
\hline
\end{tabular}


Table 36. Israel: Commodity Composition of Exports, 1997-2003 1/

\begin{tabular}{|c|c|c|c|c|c|c|c|c|c|}
\hline & \multirow[t]{2}{*}{1997} & \multirow[t]{2}{*}{1998} & \multirow[t]{2}{*}{1999} & \multirow[t]{2}{*}{2000} & \multirow[t]{2}{*}{2001} & \multirow[t]{2}{*}{2002} & \multicolumn{3}{|c|}{2003} \\
\hline & & & & & & & QI & QII & QIII \\
\hline & \multicolumn{9}{|c|}{ (In millions of U.S dollars) } \\
\hline Agricultural products & 802 & 809 & 782 & 702 & 630 & 620 & 275 & 157 & 84 \\
\hline Citrus fruit & 166 & 150 & 134 & 92 & 82 & 58 & 25 & 11 & 3 \\
\hline Other fruits and vegetables & 199 & 233 & 239 & 237 & 247 & 240 & 131 & 61 & 24 \\
\hline Other & 437 & 426 & 409 & 372 & 301 & 322 & 118 & 86 & 57 \\
\hline Industrial products (excl. diamonds) & 14,360 & 15,437 & 16,274 & 20,788 & 19,369 & 18,060 & 4,725 & 4,595 & 4,681 \\
\hline Ores and minerals & 422 & 442 & 418 & 451 & 420 & 446 & 118 & 177 & 160 \\
\hline Food (incl. Beverages and tobacco) & 527 & 476 & 439 & 444 & 427 & 429 & 125 & 124 & 128 \\
\hline Textiles (incl. Clothing and leather) & 998 & 1,064 & 1,136 & 1,144 & 1,041 & 1,003 & 261 & 233 & 250 \\
\hline Other light industry products & 1,689 & 1,665 & 1,803 & 1,880 & 1,890 & 2,006 & 513 & 528 & 559 \\
\hline Chemicals & 2,751 & 2,848 & 2,987 & 3,471 & 3,674 & 3,999 & 1,082 & 1,146 & 1,074 \\
\hline Metals, machinery and electronics & 7,932 & 8,965 & 9,506 & 13,387 & 12,038 & 10,315 & 2,625 & 2,395 & 2,519 \\
\hline Diamonds, net & 5,097 & 4,345 & 5,681 & 6,816 & 5,672 & 6,931 & 2,012 & 1,743 & 2,011 \\
\hline Other exports, n.e.s & 39 & 42 & 41 & 35 & 46 & 27 & 26 & 7 & 5 \\
\hline \multirow[t]{2}{*}{ Total, net } & 20,298 & 20,633 & 22,778 & 28,341 & 25,718 & 25,639 & 7,037 & 6,503 & 6,780 \\
\hline & \multicolumn{9}{|c|}{ (In percent) } \\
\hline Agricultural products & 4.0 & 3.9 & 3.4 & 2.5 & 2.5 & 2.4 & 3.9 & 2.4 & 1.2 \\
\hline Citrus fruit & 0.8 & 0.7 & 0.6 & 0.3 & 0.3 & 0.2 & 0.4 & 0.2 & 0.0 \\
\hline Other fruits and vegetables & 1.0 & 1.1 & 1.1 & 0.8 & 1.0 & 0.9 & 1.9 & 0.9 & 0.4 \\
\hline Other & 2.2 & 2.1 & 1.8 & 1.3 & 1.2 & 1.3 & 1.7 & 1.3 & 0.8 \\
\hline Industrial products (excl. diamonds) & 70.7 & 74.8 & 71.4 & 73.3 & 75.3 & 70.4 & 67.1 & 70.7 & 69.0 \\
\hline Ores and minerals & 2.1 & 2.1 & 1.8 & 1.6 & 1.6 & 1.7 & 1.7 & 2.7 & 2.4 \\
\hline Food (incl. Beverages and tobacco) & 2.6 & 2.3 & 1.9 & 1.6 & 1.7 & 1.7 & 1.8 & 1.9 & 1.9 \\
\hline Textiles (incl. Clothing and leather) & 4.9 & 5.2 & 5.0 & 4.0 & 4.0 & 3.9 & 3.7 & 3.6 & 3.7 \\
\hline Other light industry products & 8.3 & 8.1 & 7.9 & 6.6 & 7.3 & 7.8 & 7.3 & 8.1 & 8.2 \\
\hline Chemicals & 13.6 & 13.8 & 13.1 & 12.2 & 14.3 & 15.6 & 15.4 & 17.6 & 15.8 \\
\hline Metals, machinery and electronics & 39.1 & 43.4 & 41.7 & 47.2 & 46.8 & 40.2 & 37.3 & 36.8 & 37.1 \\
\hline Diamonds, net & 25.1 & 21.1 & 24.9 & 24.0 & 22.1 & 27.0 & 28.6 & 26.8 & 29.7 \\
\hline Other exports, n.e.s & 0.2 & 0.2 & 0.2 & 0.1 & 0.2 & 0.1 & 0.4 & 0.1 & 0.1 \\
\hline Total, net & 100.0 & 100.0 & 100.0 & 100.0 & 100.0 & 100.0 & 100.0 & 100.0 & 100.0 \\
\hline
\end{tabular}

Sources: Central Bureau of Statistics; Foreign Trade Statistics Quarterly; and Monthly Bulletin of Statistics.

1/ Excluding exports to the autonomy and administered areas. 
Table 37. Israel: Destination of Exports, 1997-2003

(In percent of total exports)

\begin{tabular}{|c|c|c|c|c|c|c|c|}
\hline & 1997 & 1998 & 1999 & 2000 & 2001 & 2002 & $20031 /$ \\
\hline Exports & 100.0 & 100.0 & 100.0 & 100.0 & 100.0 & 100.0 & 100.0 \\
\hline Europe & 37.9 & 38.0 & 36.2 & 33.2 & 32.0 & 30.7 & 32.9 \\
\hline \multicolumn{8}{|l|}{ Of which: } \\
\hline European Union & 30.5 & 31.2 & 30.2 & 27.3 & 26.3 & 24.9 & 26.6 \\
\hline Austria & 0.4 & 0.4 & 0.4 & 0.3 & 0.3 & 0.3 & 0.2 \\
\hline Belgium & 5.0 & 4.8 & 5.5 & 6.0 & 5.4 & 6.3 & 7.3 \\
\hline Denmark & 0.2 & 0.3 & 0.3 & 0.2 & 0.2 & 0.2 & 0.2 \\
\hline Finland & 0.2 & 0.2 & 0.2 & 0.2 & 0.2 & 0.3 & 0.3 \\
\hline France & 2.8 & 2.9 & 2.7 & 2.4 & 2.5 & 2.2 & 2.2 \\
\hline Germany & 4.4 & 4.9 & 4.6 & 4.8 & 4.4 & 3.5 & 3.5 \\
\hline Greece & 0.8 & 0.6 & 0.6 & 0.5 & 0.6 & 0.5 & 0.6 \\
\hline Ireland & 0.9 & 0.9 & 1.2 & 1.0 & 0.6 & 0.2 & 0.3 \\
\hline Italy & 2.8 & 3.2 & 2.9 & 2.6 & 2.7 & 2.4 & 2.5 \\
\hline Luxembourg & 0.0 & 0.0 & 0.0 & 0.0 & 0.0 & 0.0 & 0.0 \\
\hline Netherlands & 4.5 & 4.9 & 3.9 & 2.9 & 2.9 & 3.1 & 3.5 \\
\hline Portugal & 0.3 & 0.3 & 0.3 & 0.2 & 0.2 & 0.1 & 0.1 \\
\hline Spain & 1.6 & 1.8 & 1.7 & 1.4 & 1.7 & 1.4 & 1.6 \\
\hline Sweden & 0.5 & 0.5 & 0.4 & 0.5 & 0.5 & 0.4 & 0.3 \\
\hline United Kingdom & 6.2 & 5.5 & 5.4 & 4.3 & 4.2 & 4.0 & 3.9 \\
\hline EFTA countries 2/ & 2.0 & 1.9 & 1.6 & 1.7 & 1.4 & 1.5 & 1.8 \\
\hline $\begin{array}{l}\text { North, Central and South America } \\
\text { Of which: }\end{array}$ & 35.6 & 39.6 & 39.1 & 41.2 & 42.9 & 44.5 & 42.7 \\
\hline United States & 31.3 & 34.9 & 35.0 & 37.4 & 38.2 & 39.9 & 38.2 \\
\hline Asia & 19.0 & 14.3 & 16.4 & 18.5 & 18.0 & 17.3 & 17.3 \\
\hline \multicolumn{8}{|l|}{ Of which: } \\
\hline Japan & 4.6 & 3.3 & 3.4 & 2.7 & 2.8 & 2.2 & 1.9 \\
\hline Other countries & 7.5 & 8.1 & 8.3 & 7.1 & 7.1 & 7.4 & 7.1 \\
\hline
\end{tabular}

Sources: Central Bureau of Statistics; and Monthly Bulletin of Statistics.

1/ Until October.

2/ Iceland, Norway, and Switzerland. 
Table 38. Israel: Civilian Import Volume and Price Indicators, 2001-03

\begin{tabular}{|c|c|c|c|c|c|}
\hline & \multirow[t]{2}{*}{2001} & \multirow[t]{2}{*}{2002} & \multicolumn{3}{|c|}{2003} \\
\hline & & & QI & QII & QIII \\
\hline \multicolumn{6}{|l|}{ Volume indicators $1 /$} \\
\hline Consumer goods & 5.1 & -6.9 & -3.9 & 0 & 14.7 \\
\hline Nondurables & 10.9 & -0.5 & 4.9 & -3.6 & 12.3 \\
\hline Durables & -1.3 & -14.9 & -16.2 & 6.4 & 18.5 \\
\hline Input goods, net & -11.1 & 5.6 & -12.7 & 0.2 & 6.2 \\
\hline Diamonds & -20.8 & 31.5 & -29.8 & -0.9 & 16.7 \\
\hline Fuel & -0.3 & -1.9 & 4.7 & -4.5 & 7 \\
\hline Other & -9.6 & -3 & -5.5 & 2.5 & 0.2 \\
\hline Investment goods & 8.3 & -14.5 & -17.6 & 10 & -12.6 \\
\hline Machinary and equipment & 2.4 & -8.3 & -14.4 & 11.5 & -18.1 \\
\hline Total imports, net & -5.7 & -0.2 & -12.4 & 1.6 & 3.9 \\
\hline Total, excluding diamonds & -2.4 & -6.1 & -6.6 & 2.4 & 0.7 \\
\hline \multicolumn{6}{|l|}{ Price indicators $2 /$} \\
\hline Consumer goods & -1.5 & 0 & 1.4 & 1.2 & -0.4 \\
\hline Nondurables & -2.5 & -1.1 & 1.1 & 0.8 & -0.2 \\
\hline Durables & -0.2 & 1.8 & 2.6 & 1.3 & -0.8 \\
\hline Input goods, net & -1.8 & -0.9 & 4.6 & -0.2 & 1.5 \\
\hline Diamonds & 2.5 & -0.3 & 2.5 & 3.2 & 1.8 \\
\hline Fuel & -11.3 & 0.5 & 13.5 & -17.2 & 10.6 \\
\hline Other & -0.8 & -1.4 & 3.4 & 3 & -0.6 \\
\hline Investment goods & -1.1 & 2.4 & 0 & 2.3 & -0.2 \\
\hline Machinary and equipment & -1.4 & 2.3 & -0.7 & 2 & -0.1 \\
\hline Total imports, net & -1.6 & -0.2 & 3.2 & 0.5 & 1 \\
\hline Total, excluding diamonds & -2.3 & -0.5 & 3.4 & -0.2 & 0.6 \\
\hline
\end{tabular}

Sources: Central Bureau of Statistics; Foreingn Trade Statistics Quarterly; and Monthly Bulletin of Statistics.

1/ Value data deflated by paasche unit value indices.

2/ Based on data in U.S dollars. 
Table 39. Israel: Commodity Composition of Civilian Imports, 1997-2003 1/

\begin{tabular}{|c|c|c|c|c|c|c|c|c|c|}
\hline & \multirow[t]{2}{*}{1997} & \multirow[t]{2}{*}{1998} & \multirow[t]{2}{*}{1999} & \multirow[t]{2}{*}{2000} & \multirow[t]{2}{*}{2001} & \multirow[t]{2}{*}{2002} & \multicolumn{3}{|c|}{2003} \\
\hline & & & & & & & QI & QII & QIII \\
\hline & \multicolumn{9}{|c|}{ (In millions of U.S. dollars) } \\
\hline Consumer goods & 3,876 & 3,875 & 3,951 & 4,498 & 4,656 & 4,335 & 992 & 1,004 & 1,147 \\
\hline Nondurables & 2,070 & 2,146 & 2,155 & 2,363 & 2,554 & 2,513 & 615 & 598 & 670 \\
\hline Durables & 1,806 & 1,729 & 1,796 & 2,135 & 2,102 & 1,822 & 377 & 406 & 478 \\
\hline Input goods, net & 19,907 & 18,488 & 20,537 & 24,551 & 21,434 & 22,431 & 5,683 & 5,683 & 6,125 \\
\hline Diamonds & 4,779 & 3,839 & 5,456 & 6,358 & 5,164 & 6,772 & 1,609 & 1,646 & 1,955 \\
\hline Fuel & 2,260 & 1,800 & 2,109 & 3,498 & 3,095 & 3,052 & 1,000 & 791 & 936 \\
\hline Other & 12,868 & 12,849 & 12,972 & 14,695 & 13,176 & 12,607 & 3,075 & 3,246 & 3,234 \\
\hline Investment goods & 4,897 & 4,635 & 6,124 & 6,149 & 6,586 & 5,767 & 1,279 & 1,440 & 1,256 \\
\hline Machinary and equipment & 3,558 & 3,661 & 4,500 & 4,764 & 4,809 & 4,511 & 1,088 & 1,238 & 1,013 \\
\hline Total imports, net & 28,683 & 27,011 & 30,630 & 35,221 & 32,696 & 32,557 & 7,963 & 8,134 & 8,534 \\
\hline \multirow[t]{2}{*}{ Total, excluding diamonds } & 23,904 & 23,171 & 25,174 & 28,863 & 27,532 & 25,784 & 6,354 & 6,488 & 6,579 \\
\hline & \multicolumn{9}{|c|}{ (In percent) } \\
\hline Consumer goods & 13.5 & 14.3 & 12.9 & 12.8 & 14.2 & 13.3 & 12.5 & 12.3 & 13.4 \\
\hline Nondurables & 7.2 & 7.9 & 7.0 & 6.7 & 7.8 & 7.7 & 7.7 & 7.3 & 7.8 \\
\hline Durables & 6.3 & 6.4 & 5.9 & 6.1 & 6.4 & 5.6 & 4.7 & 5.0 & 5.6 \\
\hline Input goods, net & 69.4 & 68.4 & 67.1 & 69.7 & 65.6 & 68.9 & 71.4 & 69.9 & 71.8 \\
\hline Diamonds & 16.7 & 14.2 & 17.8 & 18.1 & 15.8 & 20.8 & 20.2 & 20.2 & 22.9 \\
\hline Fuel & 7.9 & 6.7 & 6.9 & 9.9 & 9.5 & 9.4 & 12.6 & 9.7 & 11.0 \\
\hline Other & 44.9 & 47.6 & 42.4 & 41.7 & 40.3 & 38.7 & 38.6 & 39.9 & 37.9 \\
\hline Investment goods & 17.1 & 17.2 & 20.0 & 17.5 & 20.1 & 17.7 & 16.1 & 17.7 & 14.7 \\
\hline Machinary and equipment & 12.4 & 13.6 & 14.7 & 13.5 & 14.7 & 13.9 & 13.7 & 15.2 & 11.9 \\
\hline Total imports, net & 100.0 & 100.0 & 100.0 & 100.0 & 100.0 & 100.0 & 100.0 & 100.0 & 100.0 \\
\hline
\end{tabular}

Sources: Central Bureau of Statistics, and Monthly Bulletin of Statistics .

1/ Excluding imports to the autonomy and administered areas. 
Table 40. Israel: Origin of Imports, 1997-2003

(In percent of total imports)

\begin{tabular}{|c|c|c|c|c|c|c|c|}
\hline & 1997 & 1998 & 1999 & 2000 & 2001 & 2002 & $20031 /$ \\
\hline Imports & 100.0 & 100.0 & 100.0 & 100.0 & 100.0 & 100.0 & 100.0 \\
\hline $\begin{array}{l}\text { Europe } \\
\text { Of which: }\end{array}$ & 59.8 & 58.1 & 56.2 & 53.0 & 52.4 & 53.0 & 53.3 \\
\hline European Union & 51.1 & 48.5 & 46.3 & 43.3 & 41.8 & 40.8 & 41.0 \\
\hline Austria & 0.5 & 0.4 & 0.3 & 0.3 & 0.4 & 0.4 & 0.4 \\
\hline Belgium & 12.4 & 10.4 & 11.3 & 9.9 & 7.9 & 9.1 & 9.1 \\
\hline Denmark & 0.5 & 0.6 & 0.4 & 0.4 & 0.5 & 0.6 & 0.4 \\
\hline Finland & 0.9 & 0.9 & 0.9 & 0.8 & 0.9 & 0.8 & 0.7 \\
\hline France & 3.8 & 4.1 & 3.9 & 3.2 & 3.8 & 3.6 & 3.5 \\
\hline Germany & 9.6 & 8.8 & 8.1 & 7.5 & 7.9 & 7.1 & 8.0 \\
\hline Greece & 0.4 & 0.5 & 0.4 & 0.4 & 0.4 & 0.4 & 0.5 \\
\hline Ireland & 0.6 & 0.8 & 0.9 & 0.9 & 0.9 & 0.8 & 0.8 \\
\hline Italy & 6.9 & 6.7 & 5.3 & 4.8 & 5.0 & 4.6 & 4.1 \\
\hline Luxembourg & 0.1 & 0.1 & 0.1 & 0.0 & 0.1 & 0.1 & 0.1 \\
\hline Netherlands & 3.8 & 4.2 & 3.8 & 4.0 & 4.1 & 3.6 & 3.5 \\
\hline Portugal & 0.4 & 0.4 & 0.3 & 0.2 & 0.2 & 0.2 & 0.2 \\
\hline Spain & 2.1 & 2.3 & 1.9 & 1.9 & 2.0 & 1.9 & 1.8 \\
\hline Sweden & 1.0 & 1.1 & 1.1 & 1.2 & 1.3 & 0.9 & 0.9 \\
\hline United Kingdom & 8.3 & 7.5 & 7.5 & 7.6 & 6.6 & 6.7 & 7.0 \\
\hline EFTA countries 2/ & 5.7 & 5.8 & 5.8 & 5.6 & 5.6 & 6.5 & 5.9 \\
\hline $\begin{array}{l}\text { North, Central and South America } \\
\text { Of which: }\end{array}$ & 20.8 & 22.1 & 22.5 & 20.5 & 22.1 & 20.9 & 17.8 \\
\hline United States & 18.7 & 19.6 & 20.3 & 18.6 & 20.1 & 18.5 & 15.8 \\
\hline Asia & 10.8 & 12.4 & 13.1 & 14.6 & 14.1 & 15.4 & 16.1 \\
\hline \multicolumn{8}{|l|}{ Of which: } \\
\hline Japan & 3.6 & 3.6 & 3.5 & 3.3 & 3.0 & 2.4 & 2.5 \\
\hline Other countries & 8.5 & 7.3 & 8.1 & 12.0 & 11.5 & 10.7 & 12.8 \\
\hline
\end{tabular}

Source: Central Bureau of Statistics, Monthly Bulletin of Statistics .

1/ Until October.

2/ Iceland, Norway, and Switzerland. 
Table 41. Israel: Capital Account Transactions, 1999-2003 1/

\begin{tabular}{|c|c|c|c|c|c|c|}
\hline & \multirow[t]{2}{*}{1999} & \multirow[t]{2}{*}{2000} & \multirow[t]{2}{*}{2001} & \multirow[t]{2}{*}{2002} & \multicolumn{2}{|c|}{2003} \\
\hline & & & & & QI & QII \\
\hline & \multicolumn{6}{|c|}{ (In millions of U.S. dollars) } \\
\hline Net financial transactions & 3,672 & 3,293 & 344 & -993 & -391 & -714 \\
\hline Net financial transactions (excl. reserve assets) & 4,601 & 4,054 & 194 & $-1,739$ & -803 & -393 \\
\hline Direct investments & 2,136 & 1,548 & 2,715 & 417 & 525 & 1,229 \\
\hline In Israel & 3,068 & 4,988 & 3,520 & 1,649 & 764 & 1,532 \\
\hline Abroad & 932 & 3,440 & 805 & 1,232 & 239 & 303 \\
\hline Portfolio investments & 1,918 & 2,229 & $-1,487$ & $-1,909$ & 183 & -474 \\
\hline In Israel & 2,598 & 5,034 & 122 & 773 & -230 & -68 \\
\hline Abroad & 680 & 2,805 & 1,609 & 2,682 & -413 & 406 \\
\hline Other investments & 696 & -387 & $-1,544$ & -438 & $-1,724$ & $-1,354$ \\
\hline In Israel & 3,492 & 1,666 & 1,166 & 1,176 & -650 & 244 \\
\hline Government & 374 & -81 & 125 & -33 & 166 & 27 \\
\hline \multicolumn{7}{|l|}{ Long term } \\
\hline Short term & -44 & 7 & -8 & 25 & 63 & -37 \\
\hline Banks & 2,385 & 1,657 & 1,271 & 117 & -751 & 128 \\
\hline \multicolumn{7}{|l|}{ Of which: } \\
\hline \multicolumn{7}{|l|}{ Other sectors } \\
\hline Long term & -67 & -116 & 104 & 121 & 78 & 149 \\
\hline Short term & 844 & 199 & -326 & 946 & -206 & -23 \\
\hline Abroad & 2,796 & 2,053 & 2,710 & 1,614 & 1,074 & 1,598 \\
\hline Government & 458 & 1 & 561 & -110 & -641 & -18 \\
\hline Banks & 758 & 1,189 & 392 & -340 & 1,093 & 1,235 \\
\hline Other sectors & 1,580 & 863 & 1,757 & 2,064 & 622 & 381 \\
\hline Financial Derivatives & -149 & 664 & 510 & 191 & 213 & 206 \\
\hline Reserve Assets & -929 & -761 & 150 & 746 & 412 & -321 \\
\hline
\end{tabular}

Sources: Central Bureau of Statistics, Monthly Bulletin of Statistics; and data provided by the Bank of Israel. 
Table 42. Israel: Indicators of External Indebtedness , 1997-2003

(In millions of U.S dollars)

\begin{tabular}{|c|c|c|c|c|c|c|c|c|}
\hline & \multirow[t]{2}{*}{1998} & \multirow[t]{2}{*}{1999} & \multirow[t]{2}{*}{2000} & \multirow[t]{2}{*}{2001} & \multirow[t]{2}{*}{2002} & \multicolumn{3}{|c|}{2003} \\
\hline & & & & & & QI & QII & QIII \\
\hline \multicolumn{9}{|l|}{ Gross external liabilities } \\
\hline Amount & 58,162 & 62,526 & 64,706 & 65,039 & 67,561 & 68,470 & 67,140 & 68,470 \\
\hline Percentage increase & 5.4 & 7.5 & 3.5 & 0.5 & 3.9 & 1.3 & $\ldots$ & $\ldots$ \\
\hline Percent of GDP & 56.3 & 60.3 & 56.4 & 57.7 & 65.2 & 62.8 & 64.5 & 65.8 \\
\hline \multicolumn{9}{|c|}{$\begin{array}{l}\text { Gross external liabilities minus } \\
\text { foreign assets of commercial banks }\end{array}$} \\
\hline Amount & 44,917 & 48,251 & 49,031 & 49,466 & 52,196 & 50,772 & 50,912 & 50,772 \\
\hline Percentage increase & 1.9 & 7.4 & 1.6 & 0.9 & 5.5 & -2.7 & $\ldots$ & $\ldots$ \\
\hline Percent of GDP & 43.4 & 46.5 & 42.7 & 43.9 & 50.3 & 46.5 & 48.9 & 48.8 \\
\hline \multicolumn{9}{|l|}{ Net external liabilities $1 /$} \\
\hline Amount & 11,295 & 7,322 & 3,713 & 210 & $-3,047$ & $-5,902$ & $-4,803$ & $-5,902$ \\
\hline Percent of GDP & 10.9 & 7.1 & 3.2 & 0.2 & -2.9 & -5.4 & -4.6 & -5.7 \\
\hline \multicolumn{9}{|l|}{ Memorandum items: } \\
\hline Foreign assets & 46,868 & 55,204 & 60,993 & 64,829 & 70,608 & 74,372 & 71,943 & 74,372 \\
\hline \multicolumn{9}{|l|}{ Of which: } \\
\hline Commercial banks & 13,246 & 14,274 & 15,675 & 15,573 & 15,365 & 17,698 & 16,228 & 17,698 \\
\hline Bank of Israel & 22,675 & 22,597 & 23,276 & 23,381 & 24,087 & 24,572 & 23,943 & 24,572 \\
\hline
\end{tabular}

Sources: Central Bureau of Statistics, Monthly Bulletin of Statistics; data provided by the Bank of Israel; and the staff calculations.

1/ Net foreign assets of commercial banks' Bank of Israel's reserves, holdings of other monetary institutions, and nonofinancial private sector. 
Table 43. Israel: Assets and Liabilities in Foreign Currency, 1997-2003 (In millions of U.S dollars)

\begin{tabular}{|c|c|c|c|c|c|c|c|c|c|}
\hline & \multirow[t]{2}{*}{1997} & \multirow[t]{2}{*}{1998} & \multirow[t]{2}{*}{1999} & \multirow[t]{2}{*}{2000} & \multirow[t]{2}{*}{2001} & \multirow[t]{2}{*}{2002} & \multicolumn{3}{|c|}{2003} \\
\hline & & & & & & & QI & QII & QIII \\
\hline 1. Gross liabilities & 55,194 & 58,162 & 62,526 & 64,706 & 65,039 & 67,561 & 68,470 & 67,140 & 68,470 \\
\hline Government & 26,187 & 27,604 & 27,778 & 27,444 & 26,865 & 27,320 & 28,098 & 27,428 & 28,098 \\
\hline Nonfinancial private sector & 11,456 & 11,367 & 13,513 & 14,577 & 14,367 & 15,720 & 16,109 & 15,828 & 16,109 \\
\hline Bank of Israel & 30 & 21 & 17 & 17 & 16 & 16 & 15 & 15 & 15 \\
\hline Banks & 17,520 & 19,170 & 21,218 & 22,668 & 23,790 & 24,505 & 24,248 & 23,869 & 24,248 \\
\hline 2. Gross assets & 40,478 & 46,868 & 55,204 & 60,993 & 64,829 & 70,608 & 74,372 & 71,943 & 74,372 \\
\hline Bank of Israel reserves & 20,333 & 22,675 & 22,597 & 23,276 & 23,381 & 24,087 & 24,572 & 23,943 & 24,572 \\
\hline Other monetary institutions & 238 & 215 & 197 & 179 & 165 & 152 & 147 & 152 & 147 \\
\hline Nonfinancial private sector & 8,561 & 10,345 & 17,481 & 21,348 & 25,301 & 30,658 & 31,842 & 31,492 & 31,842 \\
\hline Banking system & 11,104 & 13,246 & 14,274 & 15,675 & 15,573 & 15,365 & 17,698 & 16,228 & 17,698 \\
\hline 3. Net liabilities $(3=1-2)$ & 14,716 & 11,295 & 7,322 & 3,713 & 210 & $-3,047$ & $-5,902$ & $-4,803$ & $-5,902$ \\
\hline
\end{tabular}

Sources: Central Bureau of Statistics, Monthly Bulletin of Statistics; and data provided by the Bank of Israel. 
Table 44. Israel: Official Gold and Convertible

Foreign Exchange Reserves, 1997-2003

\begin{tabular}{|c|c|c|c|c|c|}
\hline & Gold 1/ & SDRs & $\begin{array}{c}\text { Reserve } \\
\text { position } \\
\text { in the Fund }\end{array}$ & $\begin{array}{c}\text { Foreign } \\
\text { exchange 2/ }\end{array}$ & Total 3/ \\
\hline & \multicolumn{5}{|c|}{ (In millions of U.S. dollars; end of period) } \\
\hline 1997 & 0.4 & 0.0 & 0.0 & 20,332 & 20,332 \\
\hline 1998 & 0.0 & 0.3 & 0.0 & 22,674 & 22,674 \\
\hline 1999 & 0.0 & 0.2 & 89.9 & 22,515 & 22,515 \\
\hline 2000 & 0.0 & 1.1 & 117.1 & 23,163 & 23,163 \\
\hline 2001 & 0.0 & 1.7 & 197.8 & 23,179 & 23,179 \\
\hline 2002 & 0.0 & 4.6 & 413.3 & 23,658 & 23,658 \\
\hline \multicolumn{6}{|l|}{2000} \\
\hline I & 0.0 & 0.3 & 88.2 & 22,677 & 22,677 \\
\hline II & 0.0 & 0.2 & 87.6 & 22,186 & 22,186 \\
\hline III & 0.0 & 0.3 & 85.0 & 22,075 & 22,075 \\
\hline IV & 0.0 & 1.1 & 117.1 & 23,163 & 23,164 \\
\hline \multicolumn{6}{|l|}{2001} \\
\hline I & 0.0 & 0.3 & 113.3 & 23,839 & 23,839 \\
\hline II & 0.0 & 0.9 & 134.1 & 23,071 & 23,072 \\
\hline III & 0.0 & 1.9 & 170.6 & 24,475 & 24,477 \\
\hline IV & 0.0 & 1.7 & 197.8 & 23,179 & 23,181 \\
\hline \multicolumn{6}{|l|}{2002} \\
\hline I & 0.0 & 1.8 & 278.1 & 23,942 & 23,943 \\
\hline II & 0.0 & 2.4 & 379.4 & 24,782 & 24,785 \\
\hline III & 0.0 & 3.3 & 401.8 & 24,218 & 24,221 \\
\hline IV & 0.0 & 4.6 & 413.1 & 23,664 & 23,669 \\
\hline \multicolumn{6}{|l|}{2003} \\
\hline I & 0.0 & 5.7 & 456.1 & 23,478 & 23,484 \\
\hline II & 0.0 & 6.8 & 476.4 & 24,082 & 24,087 \\
\hline III & 0.0 & 8.0 & 529.1 & 24,757 & 24,763 \\
\hline IV & 0.0 & 9.5 & 527.2 & 25,774 & 24,763 \\
\hline
\end{tabular}

Sources: IMF, International Financial Statistics; and data provided by the Bank of Israel.

1/ National value.

2/ At the Bank of Israel.

3/ Gold plus foreign exchange. 